Z. X. Chen, I. Langella, N. Swaminathan, M. Stöhr, W. Meier and H. Kolla, Large Eddy Simulation of a dual swirl gas turbine combustor: Flame/flow structures and stabilisation under thermoacoustically stable and unstable conditions, Combustion and Flame 203 (2019) 279-300.

The original publication is available at www.elsevier.com

https://doi.org/10.1016/j.combustflame.2019.02.013 


\title{
Large Eddy Simulation of a dual swirl gas turbine combustor: Flame/flow structures and stabilisation under thermoacoustically stable and unstable conditions
}

\author{
Zhi X. Chen ${ }^{\mathrm{a}, *}$, Ivan Langella ${ }^{\mathrm{a}, 1}$, Nedunchezhian Swaminathan ${ }^{\mathrm{a}}$, Michael Stöhr ${ }^{\mathrm{b}}$, Wolfgang Meier ${ }^{\mathrm{b}}$, Hemanth Kolla $^{\mathrm{c}}$ \\ ${ }^{a}$ Department of Engineering, University of Cambridge, Cambridge CB2 1PZ, UK \\ ${ }^{b}$ Institute of Combustion Technology, German Aerospace Center (DLR), Pfaffenwaldring 38-40, 70569 Stuttgart, Germany \\ ${ }^{c}$ Combustion Research Facility, Sandia National Laboratories, Livermore, CA 94551, USA
}

\begin{abstract}
A laboratory gas turbine model combustor with dual-swirler configuration is investigated using Large Eddy Simulation (LES) with a flamelet subgrid combustion model. Two partially premixed methane/air flames with different equivalence ratio and thermal power are simulated: one stably burning with an elongated V-shape and another undergoing pronounced thermoacoustic oscillations exhibiting a flat shape. Additionally, both flames feature a hydrodynamic instability in the form of a precessing vortex core (PVC). Detailed comparisons between experimental and LES results show that the different flow and reaction zone structures in these two flames are reproduced well. The various flow dynamics resulting from the PVC and thermoacoustic oscillations are also captured accurately in the simulation. Further analyses on the lifted swirl flame stabilisation using phase averaged statistics at the PVC frequencies reveal that the PVC-induced stagnation points provide an anchoring mechanism for both the stable and unstable flames, although in the latter case large self-excited pressure oscillations are present. It is found that the PVC is significantly influenced by these oscillations, being axially stretched and compressed at high and low pressures, respectively. However, the formation of flame leading edge due to the PVC is robust during these unstable processes and the azimuthal movement of the leading point is found to be strongly correlated with the rotation of the PVC in both flames, further confirming the vital role of the PVC in the stabilisation process of these lifted swirl flames.
\end{abstract}

Keywords: Large Eddy Simulation; Partially premixed combustion; Gas turbine model combustor; Dual Swirl; Self-excited thermoacoustic instability

\section{Introduction}

Increasingly stringent emission regulations drive the gas turbine (GT) industries towards clean (or "green") combustion technology. Fuel-lean combustion in swirling flows offers elegant flame stabilisation mechanism and a reduction in $\mathrm{NO}_{x}$ emission because of lower peak temperature of lean flames [1]. However, the flames under these conditions are prone to thermoacoustic oscillations, which are also known as combustion instabilities. This arises when the fluctuating pressure is in phase with the fluctuating heat release rate per

\footnotetext{
${ }^{*}$ Corresponding author.

Email address: zc252@cam.ac.uk (Zhi X. Chen)

${ }^{1}$ Present address: Department of Aeronautical and Automotive Engineering, Loughborough University, Loughborough, LE11 3TU, UK.
}

Preprint submitted to Journal of Combustion and Flame unit volume [2, 3]. Furthermore, if these oscillations are close to the acoustic modes of the combustor geometry, self-excited instabilities ensue. This feed-back mechanism can amplify these oscillations to amplitudes high enough to induce structural damages to the combustor [4, 5].

Significant research is devoted to understand these instabilities appearing in laboratory as well as in practical GT combustors and these studies are reviewed in [6-8]. The advances in laser diagnostics allow detailed investigation of lean swirl burners operating under GT relevant conditions and these so-called gas turbine model combustors (GTMC) are designed to obtain highly repeatable experimental observations by having well defined boundary conditions with low uncertainties. An example for such GTMC is the PRECCINSTA burner from DLR [9]11, which provided both physical in- 
sights into and model validation data for unstable lean swirl flames. Large Eddy Simulation (LES) is a natural approach to simulating unsteady processes occurring inside such GTMC and many LES studies conducted [12-15] for this burner showed good predictions for the velocity and scalar fields. These simulations assumed perfectly premixed reactant at the combustor inlet as fuel was injected far upstream inside the swirler vanes, which is valid for the stable flames simulated. However, imperfect mixing was observed in the experiments, which produced the instability for certain operating conditions [9, 11]. The influence of this imperfect mixing to capture the self-excited instability was confirmed in numerical studies by including the fuel injection from the swirler [16, 17].

The influence of imperfect mixing was also studied in experiments using another GTMC with two air swirlers and fuel injection between the two streams producing partial premixing [18,-20]. This combustor exhibited many phenomena such as flame-vortex interaction [21- 23$]$, self-excited thermoacoustic oscillations [24-27] and lean blowout dynamics [28] depending on the operating condition. Three cases were investigated in the experiments [19, 20]: a thermoacoustically stable flame, designated as flame A, an unstable flame showing self-excited thermoacoustic oscillations, called flame $\mathrm{B}$, and flame $\mathrm{C}$ exhibiting periodic blowout and reignition. These rich behaviours make this GTMC a good case for rigorous model validation. Most LES studies on this GTMC so far have focused on the stable flame A, which has a typical V-shape located in the inner shear layer (ISL) and it is stabilised by the lower stagnation point of the inner recirculation zone (IRZ). This zone and the shear layer will be highlighted while discussing the results in later sections of this paper.

See and Ihme [29] performed LES of flame A using flamelet/progress variable (FPV) approach for the subgrid combustion and showed fairly good comparisons between measured and computed velocity, temperature and major species. It is worthwhile to note that they included the upstream plenum feeding the inlet air into the inner and outer swirlers which allowed to compute the air flow split in the simulations. By contrast, the numerical works of Donini et al. [30] and Benim et al. [31] excluded the plenum by placing the inlet boundary at the inlet side of the swirler vanes to reduce the computational cost. As a result, these two studies showed quite substantial deviations between the computed and measured velocity statistics suggesting that the plenum should be included in the simulation of combustors with more than one single air passage so that the air flow split and its variation with time can be captured in the simu- lation.

As for the unstable flame $\mathrm{B}$, numerous experimental studies were conducted using the state-of-the-art measurement techniques including stereoscopic particle image velocimetry (stereo-PIV), Raman spectroscopy, laser Doppler velocimetry (LDV), $\mathrm{OH}^{*} / \mathrm{CH}^{*}$ chemiluminescence and $\mathrm{OH} / \mathrm{CH} / \mathrm{CH}_{2} \mathrm{O}$ planar laser induced fluorescence (PLIF), to investigate many interesting physics exhibited in this flame. These measurements were made using both phase-locked approach [19, 24, 25] and high repetition rate (kilo-Hertz) lasers [11, 23, 26-28]. Analysing these time and space resolved planar data have certainly improved our understanding of flame B. However, the three dimensional aspects of flow and flame features are difficult to deduce using these 2D measurements and LES becomes handy to unravel these details. To the best of authors' knowledge, so far our recent work [32] was the only numerical attempt on flame $\mathrm{B}$, which had a particular focus on the interaction between the pressure oscillations and mixing processes. Thus, more numerical effort is needed for this complex flame and the objectives of this work are:

1. To conduct LES of both flames A and B using flamelets for subgrid combustion in order to assess the abilities of the flamelet closure and LES to capture the complexities observed in these flames.

2. To offer physical insights on the stabilisation mechanisms of swirling partially premixed flames by analysing the LES data, and

3. To examine the influence of thermoacoustic oscillations on the lifted swirl flame stabilisation. To be clear, the focus here is not on thermoacoustic stability analysis but rather its influence on the anchoring mechanism of aerodynamically stabilised flames.

This paper is organised as follows. The LES formulation and subgrid combustion model are detailed in Section 2, which is followed by the description of the target burner and its numerical setup in Sections 3 and 4 . The LES results for the flame and flow structures are presented in Section 5 and the physical features of the flame stabilisation mechanism are discussed in Section 6. The concluding remarks are summarised in Section 7

\section{Modelling methodology}

The Favre-filtered transport equations for mass and momentum are solved:

$\frac{\partial \bar{\rho}}{\partial t}+\nabla \cdot(\bar{\rho} \widetilde{\boldsymbol{U}})=0, \quad$ and 
$\frac{\partial \bar{\rho} \widetilde{\boldsymbol{U}}}{\partial t}+\nabla \cdot(\bar{\rho} \widetilde{\boldsymbol{U}} \widetilde{\boldsymbol{U}})=-\nabla \bar{p}+\nabla \cdot \tau_{\mathrm{eff}}$,

where $\bar{p}$ is the modified filtered pressure, computed by solving a Poisson equation for $\bar{p}$ (combining Eqs. (1) and (2) [33]). The filtered mixture density, $\bar{\rho}$, is obtained using the ideal-gas equation of state. The effective stress tensor is modelled as $\tau_{\text {eff }}=$ $2 \bar{\rho}\left(\widetilde{v}+v_{t}\right)[\widetilde{\mathcal{S}}-(\nabla \cdot \widetilde{\boldsymbol{U}}) \mathbf{I} / 3]$ with $\widetilde{v}$ and $v_{t}$ being the filtered molecular and subgrid (SGS) eddy viscosities respectively, $\widetilde{\mathcal{S}}$ is the strain rate of $\widetilde{\boldsymbol{U}}$, and $\mathbf{I}$ is the $3 \times 3$ identity matrix. The Smagorinsky model [34] is used for the SGS eddy viscosity: $v_{t}=\left(C_{S} \Delta\right)^{2}\|\widetilde{\mathcal{S}}\|$, with the model constant $C_{S}=0.1$ and $\Delta$ is the filter width.

The compressibility effects must be included in the simulation to capture self-excited thermoacoustic oscillations [35]. Thus, the Favre-filtered transport equation for the absolute enthalpy (chemical + sensible) including the pressure effects is considered. This equation is

$\frac{\partial \bar{\rho} \widetilde{h}}{\partial t}+\nabla \cdot(\bar{\rho} \widetilde{\boldsymbol{U}} \widetilde{h})=\nabla \cdot\left[\bar{\rho}\left(\frac{\widetilde{v}}{\operatorname{Pr}}+\frac{v_{t}}{\operatorname{Pr}_{t}}\right) \nabla \widetilde{h}\right]+\frac{\overline{D p}}{D t}$,

where the laminar and turbulent Prandtl numbers, $\mathrm{Pr}$ and $\operatorname{Pr}_{t}$, are both set to be 0.7. The filtered substantial derivative of pressure is given by $\frac{\overline{D p}}{D t} \approx \frac{\partial \bar{p}}{\partial t}+\widetilde{\boldsymbol{U}} \cdot \nabla \bar{p}$.

A tabulated chemistry approach for partially premixed combustion involving a presumed SGS probability density function (PDF) is used for this work. This approach has been extended from the mixednessreactedness RANS modelling concept proposed by Bradley et al. [36, 37] using premixed flamelets, and the SGS closure models used for LES in this work have been validated for a range of test cases [38, 39]. Many other approaches sharing similar modelling concepts have also been established in the literature (for example, see reviews in Refs. [40-42] and references therein). The premixed flamelets are computed using the freely-propagating flame model in Cantera [43] with GRI Mech 3.0 for combustion kinetics. Thermochemical states of these flamelets are then tabulated as a function of filtered mixture fraction, $\widetilde{Z}$, a reaction progress variable, $\widetilde{c}$, and SGS variances of $Z$ and $c$. The turbulence-chemistry interaction is modelled via the SGS joint PDF of $Z$ and $c$. The mixture fraction is defined using Bilger mixture fraction [44]. The progress variable is defined as $c=\psi / \psi^{\mathrm{Eq}}(Z)$, where $\psi$ is the sum of $\mathrm{CO}$ and $\mathrm{CO}_{2}$ mass fractions. The superscript "Eq" denotes the burnt side value of $\psi$ in the flamelets with varying mixture fractions. The transport equations for the filtered and subgrid variances of $Z$ and $c$ are solved:

$$
\begin{aligned}
\frac{\partial \bar{\rho} \widetilde{Z}}{\partial t}+\nabla \cdot(\bar{\rho} \widetilde{\boldsymbol{U}} \widetilde{Z})=\nabla \cdot( & \left.\bar{\rho} D_{\mathrm{eff}} \nabla \widetilde{Z}\right) \\
\frac{\partial \bar{\rho} \widetilde{Z^{\prime \prime 2}}}{\partial t}+\nabla \cdot\left(\bar{\rho} \widetilde{\boldsymbol{U}} \widetilde{Z^{\prime \prime 2}}\right)= & \nabla \cdot\left(\bar{\rho} D_{\mathrm{eff}} \nabla \widetilde{Z^{\prime \prime 2}}\right)-2 \bar{\rho} \widetilde{\chi} Z, \mathrm{sgs} \\
& +2 \bar{\rho} \frac{v_{t}}{\mathrm{Sc}_{t}}|\nabla \widetilde{Z}|^{2}
\end{aligned}
$$

$\frac{\partial \bar{\rho} \widetilde{c}}{\partial t}+\nabla \cdot(\bar{\rho} \widetilde{\boldsymbol{U}} \widetilde{c})=\nabla \cdot\left(\bar{\rho} D_{\mathrm{eff}} \nabla \widetilde{c}\right)+\overline{\dot{\omega}_{c}^{*}}, \quad$ and

$$
\begin{aligned}
& \frac{\partial \bar{\rho} \widetilde{c^{\prime \prime 2}}}{\partial t}+\nabla \cdot(\left.\bar{\rho} \widetilde{\boldsymbol{U}} \widetilde{c^{\prime \prime 2}}\right)=\nabla \cdot\left(\bar{\rho} D_{\mathrm{eff}} \nabla \widetilde{c^{\prime \prime 2}}\right)-2 \bar{\rho} \widetilde{\chi}, \text {, sgs } \\
&+2 \bar{\rho} \frac{v_{t}}{\mathrm{Sc}_{t}}|\nabla \widetilde{c}|^{2}+2\left(\overline{c \dot{\omega}_{c}^{*}}-\widetilde{c} \overline{\dot{\omega}_{c}^{*}}\right),
\end{aligned}
$$

where $D_{\text {eff }}$ is the effective mixture diffusivity modelled using $\left(\widetilde{D}+v_{t} / \mathrm{Sc}_{t}\right)$ with a turbulent Schmidt number of $\mathrm{Sc}_{t}=0.4[38,45]$ and $\widetilde{D}=(\widetilde{v} / \mathrm{Sc})$ is the filtered molecular diffusivity with $\mathrm{Sc}=0.7$.

The SGS scalar dissipation rate (SDR) of mixture fraction is modelled as $\bar{\rho} \widetilde{\chi}_{Z \text {, sgs }}=C_{Z} \bar{\rho}\left(v_{t} / \Delta^{2}\right) \widetilde{Z^{\prime \prime 2}}$, where $C_{Z}$ is taken to be 2.0 [29]. This model is inadequate for the progress variable because the effects of heat release and its interaction with turbulence must be included while modelling its SDR [46-48]. Thus, a model developed and tested in earlier studies [38, 49. 51] accounting for these effects is used. This model is written as

$$
\begin{aligned}
\widetilde{\chi}_{c, \text { sgs }} & \equiv \overline{\rho D(\nabla c \cdot \nabla c)}-\bar{\rho} \widetilde{D}(\nabla \widetilde{c} \cdot \nabla \widetilde{c}) \\
& =\mathscr{F}\left[2 K_{c}^{*}(Z) \frac{S_{L}^{0}(Z)}{\delta_{L}^{0}(Z)}+\left(C_{3}-\tau C_{4} \mathrm{Da}_{\Delta}\right) \frac{2 u_{\Delta}^{\prime}}{3 \Delta}\right] \frac{c^{\prime \prime 2}}{\beta_{c}},
\end{aligned}
$$

where the subgrid velocity scale, $u_{\Delta}^{\prime}$, is modelled using a scale similarity approximation [38]. Details of the model parameters are given in Refs. [38, 39]. The model constant $\beta_{c}$ is dynamically evaluated in the LES [52].

Both flames A and B of the experiments [19, 20] selected for this work were observed to be lifted from the fuel injector suggesting partial premixing effects at the flame base [53, 54] and thus an SGS combustion model which can capture different burning modes is required. Bray et al. [55] derived the instantaneous form of Eq. (6) and showed that $\dot{\omega}_{c}^{*}$ includes the contributions from dif- 
ferent burning modes and its filtered form is written as

$$
\begin{aligned}
& \overline{\dot{\omega}}_{c}^{*}= \underbrace{{\overline{\dot{\omega}_{\psi}}}_{\psi^{\mathrm{Eq}}}}_{\text {premixed, } \bar{\omega}_{c}}+\underbrace{\rho D(\nabla Z \cdot \nabla Z) \frac{c}{\psi_{\mathrm{ep}}^{\mathrm{Eq}}} \frac{d^{2} \psi^{\mathrm{Eq}}}{d Z^{2}}}_{\text {non-premixed, }} \\
&+\underbrace{2 \rho D(\nabla Z \cdot \nabla c) \frac{1}{\psi^{\mathrm{Eq}}} \frac{d \psi^{\mathrm{Eq}}}{d Z}}_{\text {cross term, } \overline{\dot{\omega}}_{\mathrm{cdr}}},
\end{aligned}
$$

where $\overline{\dot{\omega}}_{\psi}=\overline{\dot{\omega}}_{\mathrm{CO}}+\overline{\dot{\omega}}_{\mathrm{CO} 2}$, is the filtered reaction rate of $\psi$. The three terms on the RHS, $\overline{\dot{\omega}}_{c}, \overline{\dot{\omega}}_{\text {np }}$ and $\overline{\dot{\omega}}_{\text {cdr }}$, respectively signify the contributions from premixed, non-premixed combustion modes and their interaction resulting from the cross dissipation rate. Following previous studies [38, 56, 57], $\overline{\dot{\omega}}_{\mathrm{cdr}} \approx 0$ is assumed for simplicity as the cross dissipation rate is usually small [58].

The premixed part, $\overline{\dot{\omega}}_{c}$, is modelled as

$\overline{\dot{\omega}}_{c}=\bar{\rho} \int_{0}^{1} \int_{0}^{1} \frac{\dot{\omega}_{c}(\xi, \zeta)}{\rho(\xi, \zeta)} \widetilde{P}(\xi, \zeta) d \zeta d \xi$,

where $\xi$ and $\zeta$ are the sample space variables for $Z$ and $c$ respectively. The SGS joint PDF required for Eq. (10) is to be modelled. In principle, one should consider the mutual influence between the intrinsically correlated SGS fluctuations of $Z$ and $c$ while modelling this PDF. This correlation was shown to be important for the Reynolds-Averaged Navier-Stokes (RANS) calculations [56, 57] because the fluctuations of $Z$ and $c$ inherently influence each other and this interaction is statistically significant over the sampling period, i.e., time required for the convergence of low-order statistics in RANS. However, a recent DNS study [59] showed that although the $Z$ - $c$ correlation still exists at the SGS level, it is relatively less influential on the time-averaged statistics because the portion of this correlation related to the large-scale fluctuations is resolved in LES. Thus, the subgrid correlation is not considered here for simplicity which allows to model the joint PDF using two statistically independent marginal $\beta$-PDFs of $Z$ and $c$ : $\widetilde{P}(\xi, \zeta) \approx P_{\beta}\left(\xi ; \widetilde{Z}, \widetilde{Z^{\prime \prime 2}}\right) \times P_{\beta}\left(\zeta ; \widetilde{c}, \widetilde{c^{\prime \prime 2}}\right)$. The SGS correlation effect will be investigated in a future study. Apart from the statistical independence assumption, additional error for this joint PDF approximation can originate from the presumed shape of $\beta$-distribution, and Bray et al. [60] showed that this could lead to considerable errors in the laminar flame speed if the turbulence level is small (combustion in the corrugated or wrinkled flamelets regimes). However, this error is expected to be small in highly turbulent flames such as the present case.

The non-premixed part in Eq. (9] is modelled as [38]:

$\bar{\omega}_{\mathrm{np}} \simeq \bar{\rho} \widetilde{c} \widetilde{\chi}_{Z} \int_{0}^{1} \frac{1}{\psi^{\mathrm{Eq}}(\xi)} \frac{d^{2} \psi^{\mathrm{Eq}}(\xi)}{d \xi^{2}} \widetilde{P}_{\beta}(\xi) \quad d \xi$,

where $\widetilde{\chi}_{Z}=\widetilde{D}(\nabla \widetilde{Z} \cdot \nabla \widetilde{Z})+\widetilde{\chi}_{Z \text {, sgs }}$ is the sum of the resolved and SGS (modelled) scalar dissipation rates.

The last term of Eq. (7) needs a closure and can be rewritten as $\left(\overline{c \dot{\omega}_{c}^{*}}-\widetilde{c} \overline{\dot{\omega}_{c}^{*}}\right)=$

$\left(\overline{c \dot{\omega}_{c}}-\widetilde{c} \overline{\dot{\omega}}_{c}\right)+\left(\overline{c \dot{\omega}_{\mathrm{np}}}-\widetilde{c} \overline{\dot{\omega}}_{\mathrm{np}}\right)$, where $\overline{c \dot{\omega}_{\mathrm{np}}}-\widetilde{c} \overline{\dot{\omega}}_{\mathrm{np}}=0$ following Eq. (11). The remaining term $\overline{c \dot{\omega}_{c}}$ and other thermochemical quantities such as species mass fractions, $\widetilde{Y}_{i}$, the mixture-averaged effective specific heat capacity at constant pressure and enthalpy of formation, $\widetilde{C}_{p}^{\mathrm{e}}$ and $\Delta \widetilde{h}_{f}^{0}$, are computed in a similar manner as in Eq. (10) following [56, 57]. The temperature is calculated using $\widetilde{T}=T_{0}+\left(\widetilde{h}-\Delta \widetilde{h}_{f}^{0}\right) / \widetilde{C}_{p}^{\mathrm{e}}$, where $T_{0}=298.15 \mathrm{~K}$ and $\widetilde{h}$ is obtained using Eq. (3), in which the compressible effects are included.

\section{Target burner configuration}

Figure 1 a shows a schematic of the dual-swirl burner setup [19, 20]. The dry ambient air supplied at the bottom of the plenum flows through two swirlers having the same rotational direction before exiting into the combustion chamber through concentric circular and annular nozzles of diameters 15 and $25 \mathrm{~mm}$, respectively. The fuel injector comprised 72 square nozzles $(0.5 \times$ $0.5 \mathrm{~mm}^{2}$ ) forming a ring of jets mounted on the wall between the two air nozzles. An enlarged view of this arrangement is shown in the inset of Fig. 11. These fuel nozzles were connected to an annular plenum through slightly curved ducts and pure methane was fed into this plenum through three circular inlet holes on the side wall. The exit planes of the inner air nozzle and fuel jets were $4.5 \mathrm{~mm}$ below the outer nozzle exit plane which was marked as $h=0$ for the streamwise distance (see Fig. 1). The rectangular combustion chamber was $85 \times 85 \times 110 \mathrm{~mm}^{3}$ in the $x, y$ and $h$ directions and the burnt gases flowed into the atmosphere through a duct of diameter $40 \mathrm{~mm}$.

The experimentally investigated stable flame $\mathrm{A}$ and unstable flame B are of interest here. The operating conditions of these two flames are listed in Table 1 along with the mass flow rates of air, $\dot{m}_{p}$, and fuel, $\dot{m}_{j}$, the global equivalence ratio, $\Phi_{\text {glob }}$, mixture fraction, $Z_{\text {glob }}$, swirl number and thermal power. The experimental non-reacting case of flame A with air injected through the fuel nozzles is also simulated for validating the grid 
Table 1: Cold and hot flow conditions considered

\begin{tabular}{lllllll}
\hline Case & $\Phi_{\text {glob }}$ & $Z_{\text {glob }}$ & $\dot{m}_{\mathrm{p}}(\mathrm{g} / \mathrm{s})$ & $\dot{m}_{\mathrm{j}}(\mathrm{g} / \mathrm{s})$ & $\mathrm{SN}^{*}$ & $P_{\text {therm }}(\mathrm{kW})$ \\
\hline Non-reacting & - & - & 19.74 & $1.256($ Air) & 0.9 & - \\
Flame A (stable) & 0.65 & 0.037 & 18.25 & 0.697 & 0.9 & 34.9 \\
Flame B (unstable) & 0.75 & 0.042 & 4.68 & 0.205 & 0.55 & 10.3 \\
\hline
\end{tabular}

and LES setup. The dashed lines in Fig. 19 denote the axial distance at which LDV measurements were taken for the non-reacting flow. For the reacting cases, 8 axial heights marked in Fig. 1p covering the entire combustion chamber were used. The iso-surface of $\overline{\dot{\omega}_{c}^{*}}=$ $200 \mathrm{~kg} / \mathrm{m}^{3} / \mathrm{s}$ coloured by the filtered temperature denote the computed flame surface. This is shown to help visualise the positions of the measurement locations relative to the flame. Three-component velocities were measured [21, 61] using stereoscopic PIV with highrepetition rates of 5 and $10 \mathrm{kHz}$. Laser Raman scattering was used [19, 20] to obtain the concentrations of seven major species $\left(\mathrm{CH}_{4}, \mathrm{~N}_{2}, \mathrm{O}_{2}, \mathrm{CO}_{2}, \mathrm{H}_{2} \mathrm{O}, \mathrm{CO}\right.$ and $\mathrm{H}_{2}$ ), which were then used to compute mixture fraction and temperature. The uncertainties associated with these Raman measurements were reported to be about 3 to $4 \%$ [19] except for the intermediate species, $\mathrm{CO}$ and $\mathrm{H}_{2}$, which are known to have higher uncertainties [9].

\section{Numerical Setup}

A schematic of the computational domain is shown in Fig. 2 along with the unstructured grid in the mid-y plane. As noted earlier, the air split between the inner and outer swirlers is critical to obtain the correct combustor flow field and thus the air plenum is included in the computational domain. It is also essential to have this plenum as a flow resonator when there is selfexcited oscillations. Three numerical grids are examined for the non-reacting flow to assess the mesh sensitivity and the details are given in Appendix A. Note that a uniform grid spacing of $\delta x=0.1 \mathrm{~mm}$ is used to have 5 mesh points across a fuel inlet slot $\left(0.5 \times 0.5 \mathrm{~mm}^{2}\right)$ for the $19 \mathrm{M}$ cells grid ( $\mathrm{G} 2$ case in Appendix A) used for the reacting flow results discussed in the next section.

Following previous studies [12, 16], a hemispherical domain of sufficient size is included at the combustor exit as shown in Fig. 2. The boundary conditions of this added domain are specified with atmospheric pressure. This approach avoids the difficulties in specifying a meaningful acoustic boundary condition at the combustor exit for the self-excited case. Since the majority of the turbulence in the combustion chamber is generated by the swirling flow, top-hat profiles without turbu- lence are specified for the fuel and air inlets using the measured respective mass flow rates listed in Table 1 . Ideally, the fuel plenum should also be included when there are thermoacoustic instabilities but this is not considered here as a first attempt. Adiabatic no-slip condition is employed for all the walls and Spalding's wall formula is used for the near-wall turbulence [62]. A laminar air inflow with the velocity of $0.1 \mathrm{~m} / \mathrm{s}$ is specified at the bottom of the hemispherical domain as shown in Fig. 2 .

The open-source toolbox OpenFOAM 2.3.0 is used for this study. The compressible PIMPLE solver is employed to deal with the strong coupling between pressure, velocity and scalar equations [63]. It is worth noting that the computed self-excited oscillation in flame B was found to be quite sensitive to this coupling and a stringent convergence criterion allowing for multiple PIMPLE iterations were required. Within each timestep, Eqs. (1) - 7) are solved on 3 to 5 outer loops (with 2 inner PISO loops) to obtain the correct frequency and amplitude of the oscillations in flame B. The time-step sizes for flames $\mathrm{A}$ and $\mathrm{B}$ are chosen to be $\Delta t=0.1$ and $0.3 \mu$ s based on their respective flow rates (see Table 11). The resulting maximum flow CFL number is about 0.5 near the fuel nozzle and below 0.2 over the entire domain for both cases. Once a statistically stationary state is reached, the LES data are collected for about 0.1 and $0.3 \mathrm{~s}$ for the flames $\mathrm{A}$ and $\mathrm{B}$ respectively, which correspond to 30-35 characteristic flow-throughtimes in both cases. These computations are performed on ARCHER, the UK National Supercomputer, using 1080 cores and the typical turnover time, i.e., from the start of simulation to the end of collecting statistics, is about 160 hours on the wall-clock for each case.

\section{Results and model validation}

\subsection{Reacting flow field structure and dynamics}

The computed mean axial velocity contour and streamline patterns are shown for the mid-plane in Fig. 3 for flame A. The mean flow field has a Y-shape IRZ due to the converging outlet geometry, and an outer recirculation zone (ORZ) in the corners close to injector exit 
plane. Another large-scale coherent structure often seen in swirling flows, the so-called precessing vortex core (PVC) [64], is also found in this combustor as depicted in Fig. 4 using a low dynamic pressure iso-surface of $-1425 \mathrm{~Pa}$.

The computed mean and r.m.s. values of axial, radial and swirl velocities for the two flames are compared with the measurements [19, 20] in Figs. 5 to 7] Appropriate scales are chosen for flames A and B in order to help direct comparisons and to elucidate the effect of self-excited oscillations on the flow field. The computed and measured mean axial velocity contours in a midplane of the combustor are shown in Fig. 57. The overall flow structure is well captured for both flames and the simulated IRZ and ORZ (marked by thick lines) are in reasonable agreement with the measurements. However, the width of the IRZ from $h=10$ to $30 \mathrm{~mm}$ is considerably overpredicted for flame A and the computed downstream stagnation point on the centreline appears at $h \approx 60 \mathrm{~mm}$, which is about $10 \mathrm{~mm}$ larger than that in the experiments. The same trend is also observed for flame B although the difference between the computed and measured IRZs seems to be relatively smaller. This discrepancy observed for both flames is because the flow separation in the contoured outer air nozzle lip (see enlarged view in Fig. 2a) is delayed in the computations resulting in an overestimate for the spreading angle of the swirling flow. Capturing the flow separation precisely requires a very fine near-wall mesh as discussed in Appendix A. Such a LES is computationally expensive and beyond the scope of this study, which focuses on the combustion and self-exited oscillations.

A notable difference between the two flames reported by Weigand et al. [19] is that the IRZ of flame B only reaches up to $h \approx 62 \mathrm{~mm}$, about $10 \mathrm{~mm}$ shorter than that in flame A and this is reproduced quite well in the computations, as shown in Fig. 5 a. This could be because of the longitudinal pressure oscillation present in flame B, which introduce a modulation on the inflowing streams leading to a weaker penetration compared to the stable flame A. This additional complexity posses additional challenges in capturing the flow fields in the injector near-field leading to some underprediction in the mean and r.m.s. of axial velocities for flame B compared to flame A. However, a good agreement between the simulation and measurement is observed for both flames as one moves downstream ( $h=50$ and $90 \mathrm{~mm}$ ).

The comparison for the radial velocity is presented in Fig. 6 The computed mean and r.m.s. agree well with the measurements for both flames despite some overprediction of the mean values in Fig. 6p at $h=5$ and $10 \mathrm{~mm}$ for flame $\mathrm{B}$, which is linked to the underpre- diction of the axial velocity for these positions noted in Fig. 5p. However, an excellent agreement is observed in Fig. 7between the computed and measured mean swirl velocity in both the flames for all streamwise positions. Overall, the comparisons shown above for the mean and r.m.s. of velocities are quite good for both the stable and unstable fames. This suggests that the LES framework and sub-models described in Section 2 are able to capture the complex flow and flame behaviours in the dual swirl combustor.

Meier and co-workers [21, 26, 27] performed further PIV measurements using lasers with high repetition rates of 10 and $5 \mathrm{kHz}$ for flames $\mathrm{A}$ and $\mathrm{B}$ respectively to investigate the dynamics of the coherent flow structures like the PVC and its interaction with the flame. These rep-rates were shown [21, 26] to be sufficient to resolve the unsteady motion of the PVC with the precessing frequency of about $1700 \mathrm{~Hz}$ in flame $\mathrm{A}$ and $500 \mathrm{~Hz}$ in flame B. In order to resolve the large vortices, a small measurement window close to the injector exit was chosen to have a high spatial resolution $(\sim 1.5 \mathrm{~mm})$. This window is highlighted in Fig. 8 showing the measured mean streamlines overlaid with the out-of-plane velocity contours in the mid-plane for both flames A and B. It is worth recognising that the flow field in flame B is also subject to the modulation of thermoacoustic oscillation at a frequency of about $300 \mathrm{~Hz}$ and thus it is worth performing a power spectral analysis to investigate the flow characteristics in different regions. The three representative points marked in Fig. 8 are used for the spectral analysis and these points are in the swirling jet, inner shear layer (ISL) and inner recirculation zone (IRZ) to be representative of various flow regions. Figure 9 shows the power spectra of the computed and measured axial velocities. The data were acquired for about 0.1 and $0.3 \mathrm{~s}$ from the LES of flames A and B respectively and these durations are shorter compared to $0.8 \mathrm{~s}$ in the experiments of these flames [21, 26]. The experimental results shown in Fig. 9 for flame B are similar to those reported in figure 4 of [26] where the spectra were averaged over 7 separate acquisitions totalling about $5.6 \mathrm{~s}$ for the sampling period. This suggests that a single run over a sufficiently long period is adequate to capture the significant flow and flame dynamics required for the following discussion.

The experimental data show a single peak at the PVC frequency of about $1700 \mathrm{~Hz}$ for flame A as presented in Fig. 9a for the monitoring point in the jet region. This coherent structure is predicted in the LES but with a lower frequency of about $1450 \mathrm{~Hz}$. However, the PVC frequency of about $500 \mathrm{~Hz}$ for flame B [26, 27] is captured well in the LES. The discrepancy for flame 
A could result from the less resolved velocity gradients in the ISL which dictate the PVC formation; and another possible reason is the delayed flow separation mentioned earlier leading to a larger PVC diameter (of the corkscrew) with slower rotation in the LES. As the inflow jet streams are significantly affected by the thermoacoustic oscillations, another peak at about $300 \mathrm{~Hz}$ in the experiments [26, 27] is observed for flame B. The LES captures this oscillation with a slight underprediction of $40 \mathrm{~Hz}$, which is probably caused by not including the fuel plenum in the computational domain. The spectra for the monitoring point in the ISL region shows a dominant peak for the PVC frequencies since the PVC resides in this region (see Fig. 4). The inner recirculation zone is not influenced by the PVC and thus the spectra for the IRZ monitoring point do not show the peak corresponding to the PVC frequencies but the influence of thermoacoustic oscillations can be observed at this monitoring point in Fig. 9r for flame B. As is expected, the axial velocity spectrum at this location for flame A exhibits a broad-band behaviour in both experiments and computations.

\subsection{Profiles of mixture fraction, temperature \& species}

It was observed in the experiments [19, 20] that flames A and B exhibited V-form and flat shapes, respectively. This change in flame shape is reproduced well in the LES as shown in Fig. 10 by comparing the mid-plane contours of computed mean temperature and reaction rate of progress variable between these two flames. It is seen that the conical reaction zone in flame A, which is typical for swirl flames, extends over $40 \mathrm{~mm}$ in the axial direction, nearly twice of that in flame B which shows an uncommon short and flat shape. This is caused by the additional radial fuel-air mixing pattern in flame B (see the widened stoichiometric mixture fraction iso-line), which results from the interaction between the pronounced pressure oscillations and oncoming inflows of fuel and air. This phenomenon is explained in [32] in detail and will also be discussed later in subsection 5.3. To elucidate this effect on the flame structure, the measured and computed radial profiles of flames A and B are compared in the following.

Figure 11 shows the mean and r.m.s. of mixture fraction variation across the combustor for $h=5,10,20$, 30,50 and $90 \mathrm{~mm}$. The computed mean values agree quite well with the measurements for the flame $\mathrm{A}$ at all axial locations expect a slight shift of peak position at $h=5$ and $10 \mathrm{~mm}$, which is a result of the radial shift of the jet velocity profiles due to the delayed flow separation discussed earlier in Fig. 5 For flame B considerable overprediction is observed for $h \leq 20 \mathrm{~mm}$ and the agreement improves when moving towards the downstream. Since flame B is a pulsating unstable flame with significant variation of fuel and air flow rates, the difference of $40 \mathrm{~Hz}$ between the computed and measured frequencies of the oscillation (see Fig. 9) can lead to differences in the fuel-air mixing pattern and spatial flow variations. This is reflected in the comparisons shown in Fig. 11. Also, it is worth noting that only the air plenum is included (see section 4) and the fuel plenum is not. The interaction of longitudinal pressure oscillations with fuel plenum could also play a role which will be investigated in a future study. For the r.m.s. of mixture fraction shown in Fig. $11 \mathrm{p}$, the overall agreement is quite good for both flames except for the noticeable overprediction of peak values at $h=5 \mathrm{~mm}$, which might be because of relatively low resolution used in the LES for the fuel jet exit. Also, there was some level of spatial averaging within the Raman probe $(\sim 0.6 \mathrm{~mm})$ larger than the local LES grid size at $h=5 \mathrm{~mm}$, and thus the measured r.m.s. value tends to be underestimated at this near-field location with strong mixture fraction fluctuations.

The computed radial variation of averaged and r.m.s. temperatures are compared against the measurements in Fig. 12. As shown the overall agreement is good and the substantial change in the temperature variation due to different shapes of flames A and B [19] is also captured. The averaged temperature in the IRZ $(|x|<5 \mathrm{~mm})$ located in the near field of the injector exit $(h<10 \mathrm{~mm})$ is under estimated by about $300 \mathrm{~K}$ for flame A. By contrast, the computed near-field IRZ temperature agrees well with the measurements for flame B suggesting that the influence of thermoacoustic oscillations on the reaction zones is captured accurately in the simulation. Furthermore, it was reported in the experiments [19] that the mixture reaches the burnout state before $h=20 \mathrm{~mm}$ in flame B much faster than that in flame A showing $\mathrm{CH}$ radical up to $40 \mathrm{~mm}$. This trend is also reproduced in the computations showing a flat temperature profile of about $2000 \mathrm{~K}$ by $h=20 \mathrm{~mm}$ in Fig. $12 \mathrm{p}$ for flame B whereas the flat profile is observed by $50 \mathrm{~mm}$ for flame A. The almost uniform variation of very low temperature r.m.s. values at $h=30 \mathrm{~mm}$ in flame B also supports the above observation.

For the major species $\left(\mathrm{CH}_{4}, \mathrm{O}_{2}, \mathrm{H}_{2} \mathrm{O}\right.$ and $\left.\mathrm{CO}_{2}\right)$, the level of agreement between the LES and experimental results (not shown) is similar to that for mixture fraction and temperature, which is expected for the flamelet approaches. The radial variations of computed and measured mean CO mass fractions are compared in Fig. 13 a and $13 \mathrm{~b}$ for flames $\mathrm{A}$ and $\mathrm{B}$ respectively. The error bars correspond to a $50 \%$ uncertainty [16]. It is observed 
that the trend of the measured radial and axial variations of $\mathrm{CO}$ is captured reasonably in the computations. The overestimate seen at $h=20 \mathrm{~mm}$ in Fig. $13 \mathrm{p}$ for flame B is related to the overpredicted mixture fraction (richer mixtures) close to the injector (see Fig. 11 a).

\subsection{Scatter plot of temperature vs. mixture fraction}

Scatter plot of temperature versus mixture fraction is helpful for understanding the mixing and thermochemical states of the combusting mixture at various streamwise locations inside the combustor [20]. Thus, it is used here to qualitatively compare the results obtained from LES and experiments to further examine the performance of the combustion modelling approach used for this study. This comparison also helps to reveal more insights into the flame behaviour in the stable and unstable cases. It is worth noting that the LES gives Favre-filtered quantities whereas the measured values are instantaneous which include the subgrid variations. Thus, the scatters of computed temperature tend to have a larger spread over the mixture fraction space and also a lower peak due to the filtering and density-weighting effects whilst the subgrid fluctuation is significant.

The variations of measured and computed temperature in the mixture fraction space are compared in Fig. 14 for the streamwise location of $h=5 \mathrm{~mm}$ in flames A and B. It is observed that for both flames there is a large spread of the mixture fraction ranging from 0 to 0.25 suggesting that the mixture is partially premixed at this axial location. The global value of the mixture fraction is about 0.05 and 0.06 for flames $\mathrm{A}$ and $B$ respectively. An evident difference between the experiment and simulation is seen for the mixture fraction scatters in flame $\mathrm{B}$, showing a significant population in the large values (i.e., > 0.1) with temperature above $500 \mathrm{~K}$. Despite the LES filtering effect this discrepancy is related to the overprediction of mean mixture fraction in the near field observed earlier in Fig. 11. The measured temperature variation with the mixture fraction is captured quite well in the LES and the overall agreement is good although there is an underestimate of about 300 to $400 \mathrm{~K}$ in the LES, smaller than the equilibrium values. As discussed earlier this difference originates from the strong subgrid fluctuations of temperature in the near-injector locations which cannot be resolved by the LES grid. The super-equilibrium temperature measured predominantly for IRZ and ISL regions is not seen in the computations. These super-equilibrium temperatures can result from the statistical uncertainties associated with the single-shot Raman measurements [19] and the generally higher temperature in the experiments for this near-nozzle location can also result from the hot injector metal walls preheating the unburnt gases by as much as $80 \mathrm{~K}$ for the two flames investigated [20].

The sample points in Fig. 14 are marked using different colours depending on their radial locations and five representative regions as listed in the figures. The following points are noted:

- Inner recirculation zone - IRZ $(|x|=0-2 \mathrm{~mm})$ has mainly high temperature mixture because of recirculating burnt gases and the mixtures with low temperature seen in this region are a consequence of the unsteady flame lift-off height, which is $h \approx 5$ and $4 \mathrm{~mm}$ on average for flames $\mathrm{A}$ and $\mathrm{B}$ respectively.

- Inner shear layer - ISL $(|x|=4-6 \mathrm{~mm})$ is the region where fresh and burnt gases mix rapidly and hence the majority of reactions take place in this region. As a result, the mixtures have wide range of thermochemical states yielding the largest spread (temperature ranging from 300 to $2000 \mathrm{~K}$ ). A careful interrogation of the LES data suggests the reactions occur outside the ISL in flame B because the thermoacoustic oscillations introduce flapping of the fuel jet leading to additional mixing in the radial direction, unlike in flame $\mathrm{A}$, yielding different distribution of reaction zones [32].

- Jet $(|x|=7-10 \mathrm{~mm})$ region has mostly fresh gases and thus the temperature is predominantly lower than $500 \mathrm{~K}$ with flames crossing this region occasionally.

- Outer shear layer - OSL $(|x|=14-18 \mathrm{~mm})$ contains quite different mixtures in flames A and B. This region mainly involves mixing of the air jet and recirculating burnt gases in flame A with occasional reactions. Thus, the mixtures are predominantly lean with temperature varying linearly between the air temperature $(300 \mathrm{~K})$ and the burnt temperature $(\sim 1300 \mathrm{~K})$ of the lean flammable mixture. Some unreacted rich mixtures come into this region occasionally yielding the distribution of cyan coloured points as seen in Figs. 14 and 14 . A considerable amount of reactions occur over a quite wide range of mixture fraction in this region for flame B as shown in Figs. $14 \mathrm{p}$ and $14 \mathrm{~d}$. Our recent study [32] found that this increased amount of reactions in the OSL is related to the additional radial mixing mechanism existing in the unstable case and more specifically, the fuel jet injected between two air streams (see Fig. 117) undergoes a periodic radial flapping motion towards the outer re- 
gions at the thermoacoustic frequency. This is because the two air passages in the upstream have different impedances and hence respond to the pressure oscillations differently leading to a large variation in the mass split between the two air inflows during an oscillation cycle. This variation enhances the downstream fuel-air mixing in the radial direction resulting in a shorter and flattened flame [19, 20, 32].

- Outer recirculation zone - ORZ $(|x|=21-30 \mathrm{~mm})$ : has mixtures mostly with mixture fraction values close to $Z_{\text {glob }}$ but with temperature ranging from $T_{\text {ad }}^{\mathrm{Eq}}$ to $1000 \mathrm{~K}$ in flame A as seen in Fig. 14 a. The low temperature is because of the heat loss through the walls in the experiment which is absent in the simulations because of adiabatic walls used and thus the range of temperature observed in the LES is relatively smaller as shown in Fig. 14 c. For flame B, however, the range of temperature observed is much wider (temperature as low as $500 \mathrm{~K}$ ) as seen in Fig. $14 \mathrm{p}$ and this wider temperature distribution is because of radial flapping of the fuel jet mentioned above leading to radial mixing and reaction occurring in the ORZ similar to that in the OSL. These physical processes and their effects are captured quite well in the LES as suggested by the data shown in Fig. 14d.

The variation of temperature in the mixture fraction space is shown in Fig. 15 for streamwise positions of $h=15,30,60$ and $90 \mathrm{~mm}$ for both flames A and B. It is observed that flame B reaches almost fully burnt state by about $h=30 \mathrm{~mm}$, which is significantly faster than flame A showing temperatures as low as $500 \mathrm{~K}$ at this position. The scatter of $Z$ is near $Z_{\text {glob }}$ for $h=90 \mathrm{~mm}$ suggesting that the mixing of the fuel and air streams is complete by this position. A noticeable difference between the LES and experimental results here (also in Fig. 14 is that the LES scatter is generally larger in the mixture fraction space at all streamwise positions. This is particularly evident for the two downstream locations of $h=60$ and $90 \mathrm{~mm}$, where the scatter in the LES implies an incomplete mixing as opposed to the measurement points concentrated close to the global mixture fraction. It is noted again that the LES quantities are filtered and thus expected to have a larger spread. This can be confirmed by comparing the mixture fraction PDFs (shown in embedded figures) obtained from the experiments and simulations. For both flames the LES PDFs have smaller peaks with more spread distribution, which is a typical filtering effect. Nevertheless, these variations in the evolution of $T-Z$ map inside the combustion chamber is predicted well in the LES for both flames suggesting that the modelling approach used works quite well allowing us to further analyse the LES results to gain physical insight on the mechanisms behind the stabilisation of lifted swirl flames. The influences of the PVC on the two flames and its interaction with self-excited oscillations in flame B are of specific interest here.

\section{Role of PVC in flame stabilisation $\&$ influence of self-excited oscillation}

Previous experimental studies (see Refs. [23, 26, 27]) have shown that the PVC plays an important role in the stabilisation mechanism of swirl flames in this burner. However, the experimental observations of the PVC were limited to planar measurements (e.g., PIV, PLIF). Thus, the LES data are used to gain insights into the this mechanism and its behaviour in both thermoacoustically stable and unstable flames. Figure 16 presents the $3 \mathrm{D}$ visualisation of the PVC along with contours of the filtered reaction rate in the mid-plane. The white iso-lines correspond to the mixture fraction at the lean flammability limit. The PVC in both flames is identified using the same pressure iso-surface as that used for Fig. 4. It is seen in Fig. 16 that the helical vortex core in flame $\mathrm{A}$ is about one revolution longer than that in Fig. 16 for flame B and this is similar to the experimental observation reported in [23]. In both cases, enhanced mixing of unburnt gases is observed near the fuel injector where the PVC encounters the fuel jet (marked in Fig. 16a). On the other side of the combustor axis, i.e., half a revolution downstream along the PVC, strong reactions occur since the PVC rolls the fresh gases into the IRZ. The fuel jet flapping behaviour discussed in [32] for flame B also appears here in the right half of Fig. 16 p (marked in the figure), where the PVC is not present (out of plane) and fuel jet is pushed away from the centreline. This is a consequence of the periodic variation in the mass flow split between the two air swirlers, resulting from different acoustic impedances of these swirlers felt by the pressure oscillations [32].

Regarding the flame stabilisation mechanism in the stable flame, an important finding in [23] (for thermoacoustically stable flames) was that the precessing motion of the PVC induces unsteady lower stagnation points periodically appearing at the lifted flame base, where the fresh reactants and burnt products mix directly leading to strong reactions. However, this observation was constrained by the $2 \mathrm{D}$ nature of the planar laser diagnostics and also the measurement window size (see Fig. 8), which does not include regions 
below $h=0 \mathrm{~mm}$ where the PVC strongly influences the mixing of fresh gases at fuel nozzle exit as seen in Fig. 16. To gain further insights into this, Fig. 17 shows the streamlines using the computed mid-plane velocity field overlaid on the reaction rate contours using the same snapshots shown in Fig. 16 so that it is convenient to interpret the in-plane PVC vortices using the 3D image. It can be seen in Fig. 17 that for both flames A and B, regardless of the thermoacoustic oscillations, the flame leading point seems to be near the lowest (or most-upstream) stagnation point next to the root of the PVC as marked for the chosen LES snapshot. Also, high reaction rates are found to be concentrated near the PVC vortices in the downstream regions. This is more evident for flame A in Fig. 17 a where the reaction zones are rolled up around these vortices and elongated in the ISL. By contrast, the reaction zones in flame B shown in Fig. $17 \mathrm{p}$ are more spread out radially and there is almost no reaction beyond $h=25 \mathrm{~mm}$. This difference between flames $\mathrm{A}$ and $\mathrm{B}$ is consistent with earlier observations in Figs. 12 and 15 showing a faster reaction progress in the axial direction for flame $\mathrm{B}$.

To explore the generality of the influence of the PVC on the reaction zone behaviour in these two flames, phase averages of the LES results are obtained using the computed PVC frequencies (see Fig. 9). The duration of the simulation data used span over more than 70 continuous PVC cycles for both flames and there are about 12 snapshots within each cycle. Figure 18 shows the midplane phase-averaged velocity field streamlines and filtered reaction rate contours for four typical phase angles from $0^{\circ}$ to $135^{\circ}$. Following previous studies [23, 65], a swirl strength factor is computed to identify the inplane vortices resulting from the PVC, and this factor is calculated by taking the imaginary part of the complex eigenvalues (in conjugated pairs) of the phase-averaged velocity gradient tensor [65]. The stagnation points near the flame base region are highlighted using cross markers. By comparing Fig. 18p and 18p, substantial differences can be observed between flames A and B in the phase-averaged velocity field and reaction zone behaviours.

For the stable flame A presented in Fig. 18 a, both the streamlines and reaction zone shape in the flame base region (marked in blue window) change from one phase angle to another following the movement of the large vortices. These large vortices are seen as bright white spots in the enlarged window below corresponding to high values of swirl strength factor and they are related to the most dominant large-scale structures in the flow, i.e., the PVC. At the phase angle $0^{\circ}$, there are two dominant vortical structures around the flame leading edge which is found to be well above $h=0 \mathrm{~mm}$, and the vortex on the left side starts to interact with the flame. Because of this interaction, the flame leading edge moves upstream and elongates around the left vortex (see phase angle $45^{\circ}$ ) leading to a lower lift-off height. Meanwhile, a new vortex appears on the right side of the combustor axis and then encounters the flame base at $90^{\circ}$. Finally, at phase angle $135^{\circ}$ the flame leading edge is rolled around this vortex towards the right-hand-side. The results for the remaining four phase angles from $180^{\circ}$ to $315^{\circ}$ are simply the mirrored images at the centre axis and thus not shown here. These phase-averaged reaction zone behaviours discussed above are very similar to those found experimentally in [23] for another stable flame (same equivalence ratio, lower thermal power). Furthermore, there seems to be always three stagnation points (highlighted using cross markers) located around the flame base serving as "flame anchors". The lowest stagnation point (LSP) is found between $h=-5$ and $5 \mathrm{~mm}$, and it originates periodically from the root of the PVC and then moves downstream following the PVC precession. For some phase angles (e.g., $45^{\circ}$ and $90^{\circ}$ in Fig. 18 the LSP is below $h=0$, i.e., outside the PIV window. This provides an explanation for the different LSP positions observed between the time and phase averages of the measured flow field shown in [23] because the second stagnation point (e.g. the one at $x \approx 5 \mathrm{~mm}$ at phase angle $45^{\circ}$ ) was seen as the LSP in the measurement domain.

In contrast with the flame A, there is no substantial change between different phase angles for the unstable flame B as presented in Fig. $18 \mathrm{p}$. Both the streamline pattern and reaction zone shape remain almost the same despite a slight downstream convection of the LSP resulting from the precession of the PVC. The PVC induced in-plane vortices observed earlier in Fig. $16 \mathrm{p}$ and $17 \mathrm{p}$ are not visible here in the phase-averaged streamlines, and no clear structure of the PVC can be identified in the contours of swirl strength factor. This is because the thermoacoustic oscillations at the computed frequency of about $260 \mathrm{~Hz}$ periodically interfere the mean flow and the PVC. Specifically, for two instants with the same phase angle of the PVC, the behaviour of the PVC (e.g., position and strength) and its interaction with the flame can vary significantly if those two instants are in different phases of a thermoacoustic cycle. Steinberg and co-workers [27] showed in a previous experimental study that in flame B the PVC undergoes periodic axial movements at the thermoacoustic frequency. These variations cancel out when the phase averaging is applied only based on the PVC frequency as seen in Fig. 18 . 
To demonstrate the effect of thermoacoustic oscillation on the PVC, the relative phase variation of the PVC to the pressure fluctuation phase needs to be identified. Figure $[19 \mathrm{a}$ presents the normalised temporal variation of the pressure inside the combustion chamber for the time period between 70 and $78 \mathrm{~ms}$, which spans about two thermoacoustic cycles. Here the phase of the PVC is defined such that the orientation indicator is 1 when the PVC crosses the $h=0$ line on the left side of the chosen mid-plane shown in Figs. $19 \mathrm{~b}$ and 19 . This particular time window is selected because the PVC orientation relative to the plane is the same (indicator $=1$ ) for the two instants of $t=72.6$ and $74.64 \mathrm{~ms}$, but the chamber pressure is the maximum and minimum respectively within one thermoacoustic cycle. In Figs $19 \mathrm{p}$ and 19. the instantaneous reaction rate contours (in green colours) are overlaid on the pressure along with the isoline for the mixture fraction of 0.07 to mark the reaction zone and fuel distributions. It can be seen in both figures that the PVC induced flame roll-up and enhanced mixing behaviours are quite similar to those discussed earlier in Fig. 16p despite the pressure difference. However, the axial distance between the two PVC vortices at $t=72.6 \mathrm{~ms}$ is much longer than $t=74.64 \mathrm{~ms}$, being about 11 and $4 \mathrm{~mm}$, respectively. This is consistent with the experimental study [27] which showed that the PVC axially extends at high pressure and retracts at low pressure. Similar behaviours are also observed in other time periods (not shown). Apart from this axial motion, another effect of the pressure oscillation found here is that the PVC root residing in the inner air nozzle contracts and expands radially at high and low pressures respectively. Occasionally, in few cycles with temporarily large oscillations the root vanishes completely and recovers later when the pressure fluctuation becomes normal again. This additional PVC motion could not be seen in the experiments due to the limitation of optical access into the nozzles. Ideally, one would need to perform doubly phase-averaging techniques to separate these two dynamic processes and the present simulation runtime is insufficient to obtain statistically meaningful results using such techniques. Thus, 3D mode composition methods such as dynamic mode decomposition (DMD) are the appropriate tool to reveal further insights on the PVC behaviours [66]. This analysis is beyond the scope of this study and will be investigated in future.

It is of interest to study the flame leading edge movement in the azimuthal direction as the PVC precesses about the centre-axis. Following previous studies [38, 57], the most-upstream-point of the temperature $1200 \mathrm{~K}$ iso-surface above the fuel injectors $(|x|<$ $10 \mathrm{~mm}$ ) is defined as the flame leading point as illus- trated in Fig. 20a). By applying FFT on the time series of the azimuthal position fluctuations, $\theta^{\prime}$, the spectral behaviour of the azimuthal movement of this flame leading point is shown in Figs. $20 \mathrm{p}$ and $20 \mathrm{c}$ for flames A and $\mathrm{B}$ respectively. It can be clearly seen that a pronounced single peak appears at the PVC frequency for both flames. This lends further support to the viewpoint that the PVC provides the essential mechanism for the lifted flame stabilisation and the flame leading point follows the movement of the PVC in the azimuthal direction. It is quite remarkable to see that the azimuthal movement of the flame leading point is still strongly correlated with the PVC frequency in flame B, even though the PVC is significantly modulated by the selfexcited oscillations and not visible in the phase averages shown in Fig. 18p. This can also be seen in Figs. 19p and 19 where the flame leading edge seems to follow the in-plane PVC vortices even though the PVC itself is undergoing a large axial movement between the two snapshot presented. Therefore, these results imply that the stabilisation mechanism based on the flame-PVC interaction is quite robust for lifted swirl flames with strong turbulent fluctuations and even in the presence of large flow pulsations induced by thermoacoustic oscillations.

\section{Conclusions}

This paper presents a Large Eddy Simulation (LES) study of partially premixed methane-air flames in a laboratory gas turbine model combustor with dual swirlers. Two flames, one stably burning with strong vortexflame interaction, and another unstable flame showing pronounced self-excited thermoacoustic instability, are simulated using a revised flamelet approach. The simulation results are compared with an extensive experimental dataset [19,-21, 26] for model validation. For both flames considered, the results show good overall agreement between the computed and measured flow and flame structures suggesting that the modelling approach used is robust. The swirling jet spread angle from the centreline is overpredicted considerably in both cases. This is because of the difficulties in accurately capturing the flow separation from the contoured wall of the outer air nozzle in LES. Different dynamic behaviours of the flow field between the stable and unstable flames resulting from the interplay among the lifted swirl flame, precessing vortex core (PVC) and thermoacoustic oscillations are captured well. The predicted frequency of the self-excited oscillations is slightly lower than the measured value, which is possibly due to the absence of the fuel plenum in the simu- 
lation. The investigation on this is underway and results will be present in a future study.

The LES results are further analysed to gain physical insights into the stabilisation mechanism of these lifted swirl flames. It is shown that in both stable and unstable flames the PVC induced stagnation points form an anchoring mechanism for the lifted flame base to stabilise in a low-velocity region, where the fresh reactants and burnt product mix leading to strong reactions. Moreover, the flame leading point is always found to be close to the lowest stagnation point. Phase-averaging is applied to investigate these effects at different PVC phase angles. The results for the stable flame show similar behaviours of the stagnation points as those found using a single snapshot, while it is not so for the unstable flame. This is because in presence of strong thermoacoustic oscillations the PVC motion is significantly influenced by the large flow pulsations at a different frequency. It is found that the PVC exhibits a sponge-like motion during a thermoacoustic cycle, i.e., axial extension and radial contraction at high pressure, and vice versa at low pressure. However, the azimuthal movement of the flame leading edge still follows the PVC precession in this unstable flame, similar to that found in the stable flame and this further confirms the key role of the PVC in the swirl flame stabilisation process even under different thermoacoustic conditions.

\section{Acknowledgement}

ZXC and NS acknowledge the support of Mitsubishi Heavy Industries, Takasago, Japan. This work used the ARCHER UK National Supercomputing Service (http://www.archer.ac.uk) using computing time provided by EPSRC under the UKCTRF (e305).

\section{Appendix A. Assessment of mesh sensitivity}

The non-reacting case in Table 1 is studied to assess the mesh dependency using the three grid sizes detailed in Table A.1. Note that in the regions other than those listed in the table, e.g., the upstream air plenum and downstream atmospheric far field, the mesh cell sizes are the same for all three grids. In the cold flow experiments [67], radial profiles of three velocity components (axial, radial and swirl) were measured at the five streamwise locations marked in Fig. 1. These measurements, both time-averaged and r.m.s. values, are compared with the LES results in Fig. A.1.

There is a strong grid sensitivity observed between G1 and G2 for all three components whereas little difference is seen between G2 and G3, both giving much
Table A.1: Typical grid sizes (unit $[\mathrm{mm}]$ ) in the computational domain.

\begin{tabular}{llll}
\hline Mesh zone & G1 & G2 & G3 \\
\hline Fuel nozzle & 0.1 & 0.1 & 0.1 \\
Swirlers & 0.5 & 0.3 & 0.2 \\
Shear layers & 1 & 0.5 & 0.4 \\
Rest of combustion chamber & 2 & 1 & 0.8 \\
\hline Total No. of cells [million] & 12 & 15 & 20 \\
\hline
\end{tabular}

improved agreement with the experimental data compared to G1. This improvement obtained using G2 and G3 is more evident for the axial and radial velocities in Figs. A.1 and A.1 showing a better prediction of the peak location at about $|x|=10 \mathrm{~mm}$ for $h=2.5 \mathrm{~mm}$ and this peak shifts outwardly while one moves downstream.

It is of particular interest for this configuration to take additional care while assessing the mesh quality in the outer air nozzle region (marked using a red box in Fig. 3). This is because the contoured outer lip geometry causes a sudden expansion of cross-sectional area in the streamwise direction and as a consequence, the flow separates from the nozzle outer wall forming a small local recirculation zone [67] as demonstrated in Fig. A.2a.

In order to capture this phenomenon, a near-wall mesh refinement is applied along this contoured surface for grid G2 and G3 to have a good wall resolution with $y^{+} \approx 5$. Although this is still insufficient to fully resolve the boundary layer and thus predict the flow separation accurately, which would require $y^{+}<<1$, the flow field is captured reasonably well when compared with experimental data in Fig. A.1

Based on these results, the grid G2 is adopted for the reacting flow simulations presented in this study. Grid cells near the fuel nozzle are refined having a uniform spacing of $\delta x=0.1 \mathrm{~mm}$. The final grid consists of about 19 million tetrahedral cells. As noted earlier in Table A.1, the typical cell size in the flame region is $0.5 \mathrm{~mm}$ and this corresponds to a normalised filter size of $\Delta^{+} \approx 0.8$, where $\Delta^{+}=\Delta x /\left(\delta_{L}^{0}\right)_{\text {st }}$ with $\left(\delta_{L}^{0}\right)_{\text {st }}$ being the thermal thickness of the laminar premixed stoichiometric methane-air flame. Figure A.3 presents a typical histogram of $\Delta^{+}$within the flame region for a given snapshot of flame A. It can be seen that the LES filter width in the flame area is of the order of the laminar flame thickness. Hence, the typical numerical grid used for this study does not resolve the flame front and the combustion processes occur at the subgrid scales, which are modelled using the methodology described in Section 2 . 


\section{References}

[1] A.H. Lefebvre, D.R. Ballal, Gas turbine combustion: alternative fuels and emissions, CRC Press, Boca Raton, USA, 2010.

[2] J. Rayleigh, The explanation of certain acoustic phenomena, Nature 18 (1878) 319-321.

[3] J. Rayleigh, The Theory of Sound, Vol. II, Dover, New York, 1945.

[4] S. Candel, Combustion dynamics and control: Progress and challenges, Proc. Combust. Inst. 29 (2002) 1-28.

[5] T.C. Lieuwen, V. Yang (Eds.), Combustion instabilities in gas turbine engines: Operational experience, fundamental mechanisms, and modeling, AIAA Inc., Reston, VA, 2006.

[6] Y. Huang, V. Yang, Dynamics and stability of lean-premixed swirl-stabilized combustion, Prog. Energy Combust. Sci. 35 (4) (2009) 293-364.

[7] T.C. Lieuwen, Unsteady combustor physics, Cambridge University Press, 2012.

[8] T. Poinsot, Prediction and control of combustion instabilities in real engines, Proc. Combust. Inst. 36 (2017) 1-28.

[9] W. Meier, P. Weigand, X.R. Duan, R. Giezendanner-Thoben, Detailed characterization of the dynamics of thermoacoustic pulsations in a lean premixed swirl flame, Combust. Flame 150 (2007) 2-26.

[10] V. Caux-Brisebois, A.M. Steinberg, C.M. Arndt, W. Meier, Thermo-acoustic velocity coupling in a swirl stabilized gas turbine model combustor, Combust. Flame 161 (2014) 3166-3180.

[11] M. Stöhr, Z. Yin, W. Meier, Interaction between velocity fluctuations and equivalence ratio fluctuations during thermoacoustic oscillations in a partially premixed swirl combustor, Proc. Combust. Inst. 36 (2017) 3907-3915.

[12] S. Roux, G. Lartigue, T. Poinsot, U. Meier, C. Bérat, Studies of mean and unsteady flow in a swirled combustor using experiments, acoustic analysis, and large eddy simulations, Combust. Flame 141 (2005) 40-54.

[13] J. Galpin, A. Naudin, L. Vervisch, C. Angelberger, O. Colin, P. Domingo, Large-eddy simulation of a fuel-lean premixed turbulent swirl-burner, Combust. Flame 155 (2008) 247-266.

[14] B. Fiorina, R. Vicquelin, P. Auzillon, N. Darabiha, O. Gicquel, D. Veynante, A filtered tabulated chemistry model for LES of premixed combustion, Combust. Flame 157 (2010) 465-475.

[15] V. Moureau, P. Domingo, L. Vervisch, From large-eddy simulation to direct numerical simulation of a lean premixed swirl flame: Filtered laminar flame-pdf modeling, Combust. Flame 158 (2011) 1340-1357.

[16] B. Franzelli, E. Riber, L.Y.M. Gicquel, T. Poinsot, Large eddy simulation of combustion instabilities in a lean partially premixed swirled flame, Combust. Flame 159 (2012) 621-637.

[17] J.-M. Lourier, M. Stöhr, B. Noll, B. Werner, A. Fiolitakis, Scale Adaptive Simulation of a thermoacoustic instability in partially premixed lean swirl combustor, Combust. Flame 183 (2017) 343-357.

[18] W. Meier, X.R. Duan, P. Weigand, Reaction zone structures and mixing characteristics of partially premixed swirling $\mathrm{CH} 4 /$ air flames in a gas turbine model combustor, Proc. Combust. Inst. 30 (2005) 835-842.

[19] P. Weigand, W. Meier, X.R. Duan, W. Stricker, M. Aigner, Investigations of swirl flames in a gas turbine model combustor i. flow field, structures, temperature, and species distributions, Combust. Flame 144 (2006) 205-224.

[20] W. Meier, X.R. Duan, P. Weigand, Investigations of swirl flames in a gas turbine model combustor ii. turbulence-chemistry interactions, Combust. Flame 144 (2006) 225-236.

[21] M. Stöhr, C.M. Arndt, W. Meier, Effects of Damköhler number on vortex-flame interaction in a gas turbine model combustor, Proc. Combust. Inst. 34 (2013) 3107-3115.

[22] M. Stöhr, C.M. Arndt, W. Meier, Transient effects of fuel-air mixing in a partially-premixed turbulent swirl flame, Proc. Combust. Inst. 35 (2015) 3327-3335.

[23] M. Stöhr, I. Boxx, C.D. Carter, W. Meier, Experimental study of vortex-flame interaction in a gas turbine model combustor, Combust. Flame 159 (2012) 2636-2649.

[24] R. Giezendanner, P. Weigand, X.R. Duan, W. Meier, U. Meier, M. Aigner, B. Lehmann, Laser-based investigations of periodic combustion instabilities in a gas turbine model combustor, J. Eng. Gas Turbine Power 127 (2005) 492-496.

[25] X.R. Duan, W. Meier, P. Weigand, B. Lehmann, Phase-resolved laser Raman scattering and laser Doppler velocimetry applied to periodic instabilities in a gas turbine model combustor, Applied Physics B: Lasers and Optics 80 (3) (2005) 389-396.

[26] I. Boxx, M. Stöhr, C. Carter, W. Meier, Temporally resolved planar measurements of transient phenomena in a partially premixed swirl flame in a gas turbine model combustor, Combust. Flame 157 (2010) 1510-1525.

[27] A.M. Steinberg, I. Boxx, M. Stöhr, C.D. Carter, W. Meier, Flow-flame interactions causing acoustically coupled heat release fluctuations in a thermo-acoustically unstable gas turbine model combustor, Combust. Flame 157 (2010) 2250-2266.

[28] M. Stöhr, I. Boxx, C. Carter, W. Meier, Dynamics of lean blowout of a swirl-stabilized flame in a gas turbine model combustor, Proc. Combust. Inst. 33 (2011) 2953-2960.

[29] Y.C. See, M. Ihme, Large eddy simulation of a partiallypremixed gas turbine model combustor, Proc. Combust. Inst. 35 (2015) 1225-1234.

[30] A. Donini, R.J.M. Bastiaans, J.A. van Oijen, L.P.H. de Goey, A 5-D Implementation of FGM for the Large Eddy Simulation of a Stratified Swirled Flame with Heat Loss in a Gas Turbine Combustor, Flow Turbul. Combust. 98 (3) (2017) 887-922.

[31] A.C. Benim, S. Iqbal, W. Meier, F. Joos, A. Wiedermann, Numerical investigation of turbulent swirling flames with validation in a gas turbine model combustor, Appl. Therm. Eng. 110 (2017) 202-212.

[32] Z.X. Chen, N. Swaminathan, M. Stöhr, W. Meier, Interaction between self-excited oscillations and fuel-air mixing in a dual swirl combustor, Proc. Combust. Inst. 37 (2019) 2325-2333.

[33] S.B. Pope, Turbulent Flows, Cambridge University Press, 2000.

[34] J. Smagorinsky, General circulation experiments with the primitive equations, Mon. Weather Rev. 91 (3) (1963) 99-164.

[35] T. Poinsot, D. Veynante, Theoretical and Numerical Combustion, 2nd Edition, Edwards, 2005.

[36] D. Bradley, P.H. Gaskell, A.K.C. Lau, A mixedness-reactedness flamelet model for turbulent diffusion flames, Proc. Combust. Inst. 23 (1990) 685-692.

[37] D. Bradley, P.H. Gaskell, X.J. Gu, The mathematical modeling of liftoff and blowoff of turbulent non-premixed methane jet flames at high strain rates, Proc. Combust. Inst. 27 (1998) 1199-1206.

[38] Z. Chen, S. Ruan, N. Swaminathan, Large Eddy Simulation of flame edge evolution in a spark-ignited methane-air jet, Proc. Combust. Inst. 36 (2017) 1645-1652.

[39] I. Langella, Z.X. Chen, N. Swaminathan, S.K. Sadasivuni, Large-Eddy Simulation of reacting flows in industrial gas turbine combustor, J. Propul. Power 34 (2018) 1269-1284.

[40] D. Veynante, L. Vervisch, Turbulent combustion modeling, Prog. Energy Combust. Sci. 28 (2002) 193-266.

[41] J. Janicka, A. Sadiki, Large eddy simulation of turbulent combustion systems, Proc. Combust. Inst. 30 (2005) 537-547.

[42] J.A. van Oijen, A. Donini, R.J.M. Bastiaans, J.H.M. ten Thije Boonkkamp, L.P.H. de Goey, State-of-the-art in premixed com- 
bustion modeling using flamelet generated manifolds, Prog. Energy Combust. Sci. 57 (2016) 30-74.

[43] D.G. Goodwin, H.K. Moffat, R.L. Speth, Cantera: An Objectoriented Software Toolkit for Chemical Kinetics, Thermodynamics, and Transport Processes, http://www.cantera.org (2017).

[44] R.W. Bilger, Structure of diffusion flames, Combust. Sci. Technol. 13 (1976) 155-170.

[45] H. Pitsch, H. Steiner, Large-eddy simulation of a turbulent piloted methane/air diffusion flame (sandia flame D), Phys. Fluids 12 (2000) 2541

[46] N. Swaminathan, K.N.C. Bray, Effect of dilatation on scalar dissipation in turbulent premixed flames, Combust. Flame 143 (4) (2005) 549-565.

[47] N. Swaminathan, R.W. Grout, Interaction of turbulence and scalar fields in premixed flames, Phys. Fluids 18 (2006) 045102.

[48] N. Chakraborty, J.W. Rogerson, N. Swaminathan, A priori assessment of closures for scalar dissipation rate transport in turbulent premixed flames using direct numerical simulation, Phys. Fluids 20 (2008) 045106.

[49] T.D. Dunstan, Y. Minamoto, N. Chakraborty, N. Swaminathan, Scalar dissipation rate modelling for large eddy simulation of turbulent premixed flames, Proc. Combust. Inst. 34 (2013) 1193-1201.

[50] I. Langella, N. Swaminathan, R.W. Pitz, Application of unstrained flamelet SGS closure for multi-regime premixed combustion, Combust. Flame 173 (2016) 161-178.

[51] I. Langella, N. Swaminathan, Unstrained and strained flamelets for LES of premixed combustion, Combust. Theory Model. 20 (2016) 410-440.

[52] I. Langella, N. Swaminathan, Y. Gao, N. Chakraborty, Assessment of dynamic closure for premixed combustion large eddy simulation, Combust. Theory Model. 19 (5) (2015) 628-656.

[53] N. Peters, Turbulent Combustion, Cambridge University Press, 2000.

[54] A.R. Masri, Partial premixing and stratification in turbulent flame, Proc. Combust. Inst. 35 (2015) 1115-1136.

[55] K.N.C. Bray, P. Domingo, L. Vervisch, Role of the progress variable in models for partially premixed turbulent combustion, Combust. Flame 141 (4) (2005) 431-437.

[56] S. Ruan, N. Swaminathan, O.R. Darbyshire, Modelling of turbulent lifted jet flames using flamelets: a priori assessment and a posteriori validation, Combust. Theory Model. 18 (2) (2014) 295-329.

[57] Z. Chen, S. Ruan, N. Swaminathan, Simulation of turbulent lifted methane jet flames: effects of air-dilution and transient flame propagation, Combust. Flame 162 (2015) 703-716.

[58] S. Ruan, N. Swaminathan, K.N.C. Bray, Y. Mizobuchi, T. Takeno, Scalar and its dissipation in the near field of turbulent lifted jet flame, Combust. Flame 159 (2012) 591-608.

[59] Z.X. Chen, N.A.K. Doan, S. Ruan, I. Langella, N. Swaminathan, A priori investigation of subgrid correlation of mixture fraction and progress variable in partially premixed flames, Combust. Theory Model. 22 (2018) 862-882.

[60] K.N.C. Bray, M. Champion, P.A. Libby, N. Swaminathan, Finite rate chemistry and presumed PDF models for premixed turbulent combustion, Combust. Flame 146 (4) (2006) 665-673.

[61] M. Stöhr, R. Sadanandan, W. Meier, Experimental study of unsteady flame structures of an oscillating swirl flame in a gas turbine model combustor, Proc. Combust. Inst. 32 (2009) 29252932.

[62] D.B. Spalding, A single formula for the "Law of the Wall", J. Appl. Mech 28 (1961) 455-458.

[63] Z. Chen, Simulation of Partially Premixed Turbulent Flames, Ph.D. thesis, University of Cambridge (2016).
[64] N. Syred, A review of oscillation mechanisms and the role of the precessing vortex core (PVC) in swirl combustion systems, Prog. Energy Combust. Sci. 32 (2006) 93-161.

[65] J. Zhou, R.J. Adrian, S. Balachandar, T.M. Kendall, Mechanisms for generating coherent packets of hairpin vortices in channel flow, J. Fluid Mech. 387 (1999) 353-396.

[66] S. Roy, T. Yi, N. Jiang, G.H. Gunaratne, I. Chterev, B. Emerson, T. Lieuwen, A.W. Caswell, J.R. Gord, Dynamics of robust structures in turbulent swirling reacting flows, J. Fluid Mech. 816 (2017) 554-585.

[67] A. Widenhorn, B. Noll, M. Aigner, Numerical study of a nonreacting turbulent flow in a gas turbine model combustor, AIAA Paper 647 (2009) 1-17. 

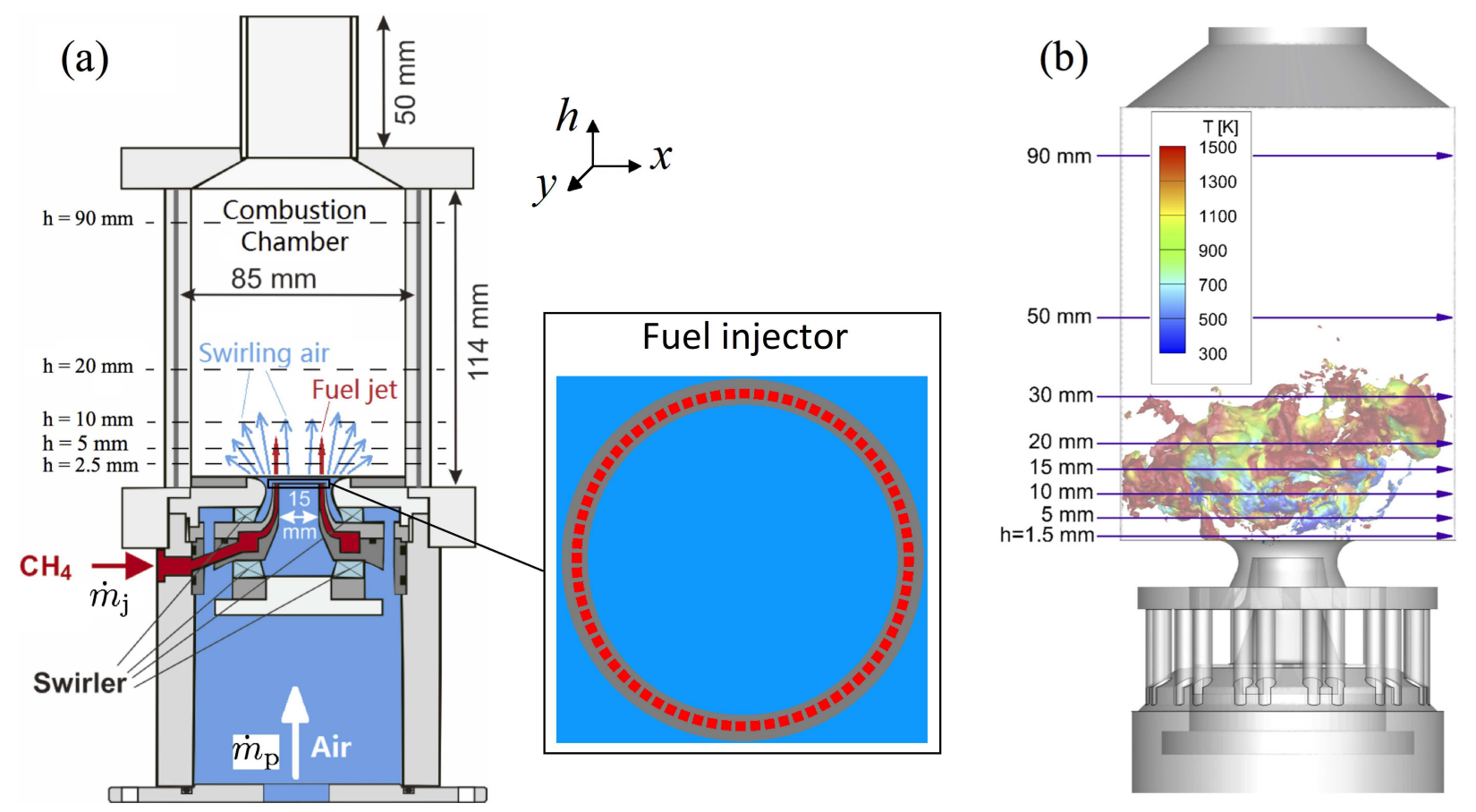

Fig. 1: The dual swirl combustor: (a) schematic of the experimental setup and (b) typical flame surface marked using $\overline{\dot{\omega}_{c}^{*}}=200 \mathrm{~kg} / \mathrm{m}^{3} / \mathrm{s}$, coloured by temperature.

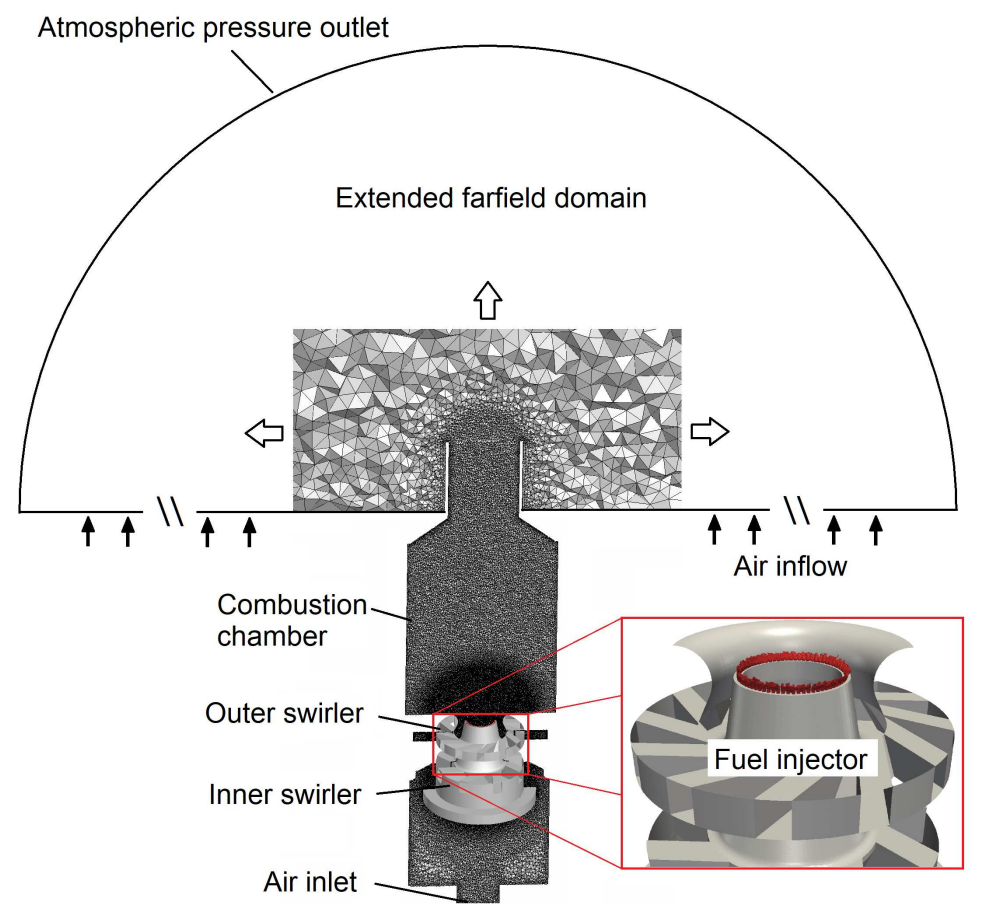

Fig. 2: Schematic of the computational domain and mesh resolution on the mid-plane. Enlarged view: fuel injection through the 72 square nozzles visualised using the $\widetilde{Z}=0.7$ iso-surface. 


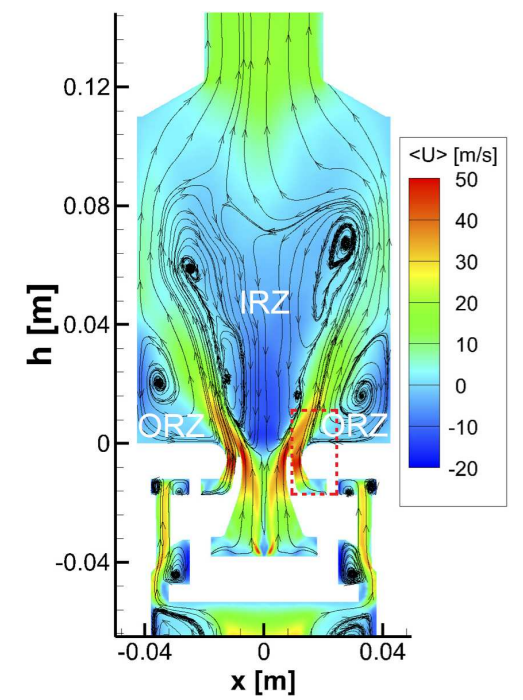

Fig. 3: Mid-plane contour of computed mean axial velocity with streamlines for flame A. (The marked area is used for later discussion in Appendix A.)

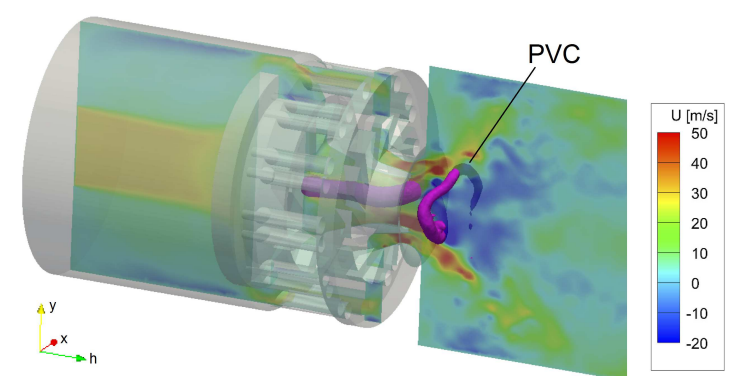

Fig. 4: 3D visualisation of the computed precessing vortex core (PVC) for flame A along with a mid-plane axial velocity snapshot. 


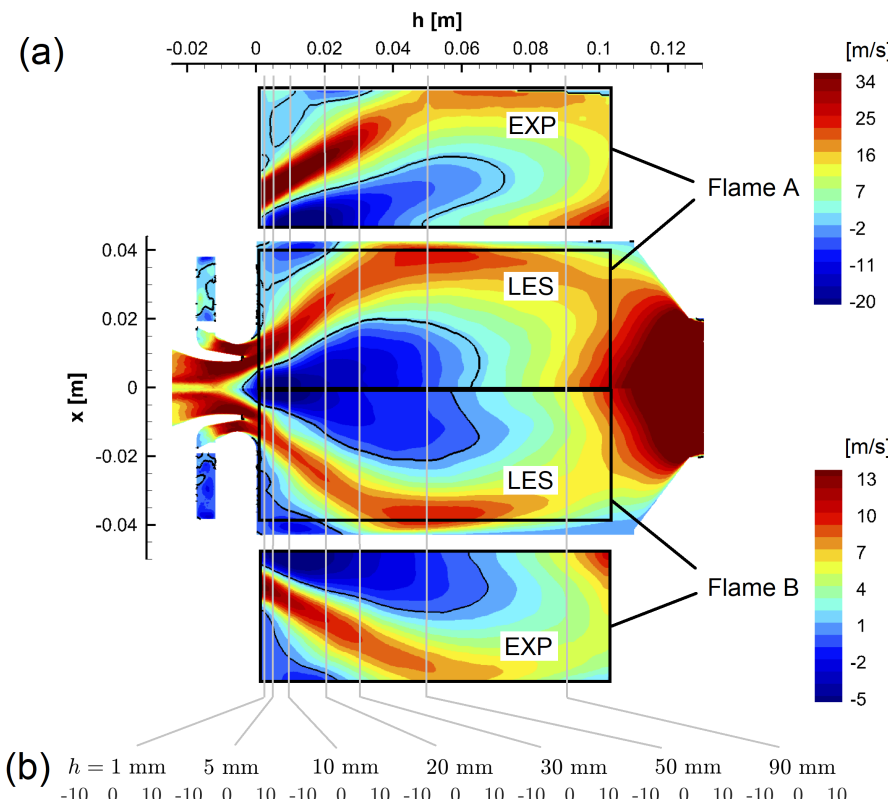

(b) $h=1 \mathrm{~mm} \quad 5 \mathrm{~mm} \quad 10 \mathrm{~mm} \quad 20 \mathrm{~mm} \quad 30 \mathrm{~mm} \quad 50 \mathrm{~mm} \quad 90 \mathrm{~mm}$
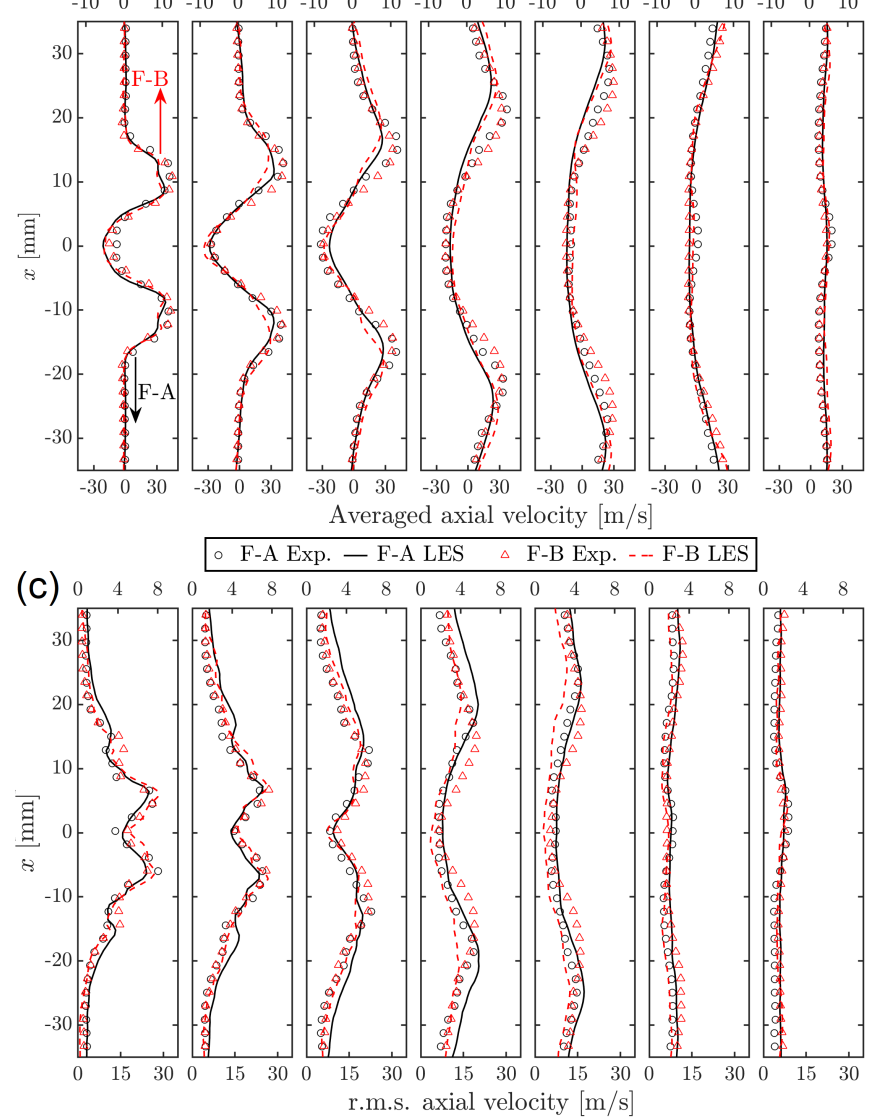

Fig. 5: Comparison of axial velocity for flames A and B: (a) mid-plane contour with the recirculation zones marked using thick lines, (b) averaged and (c) r.m.s profiles at different axial locations. Symbols: experimental data. Lines: LES results. 


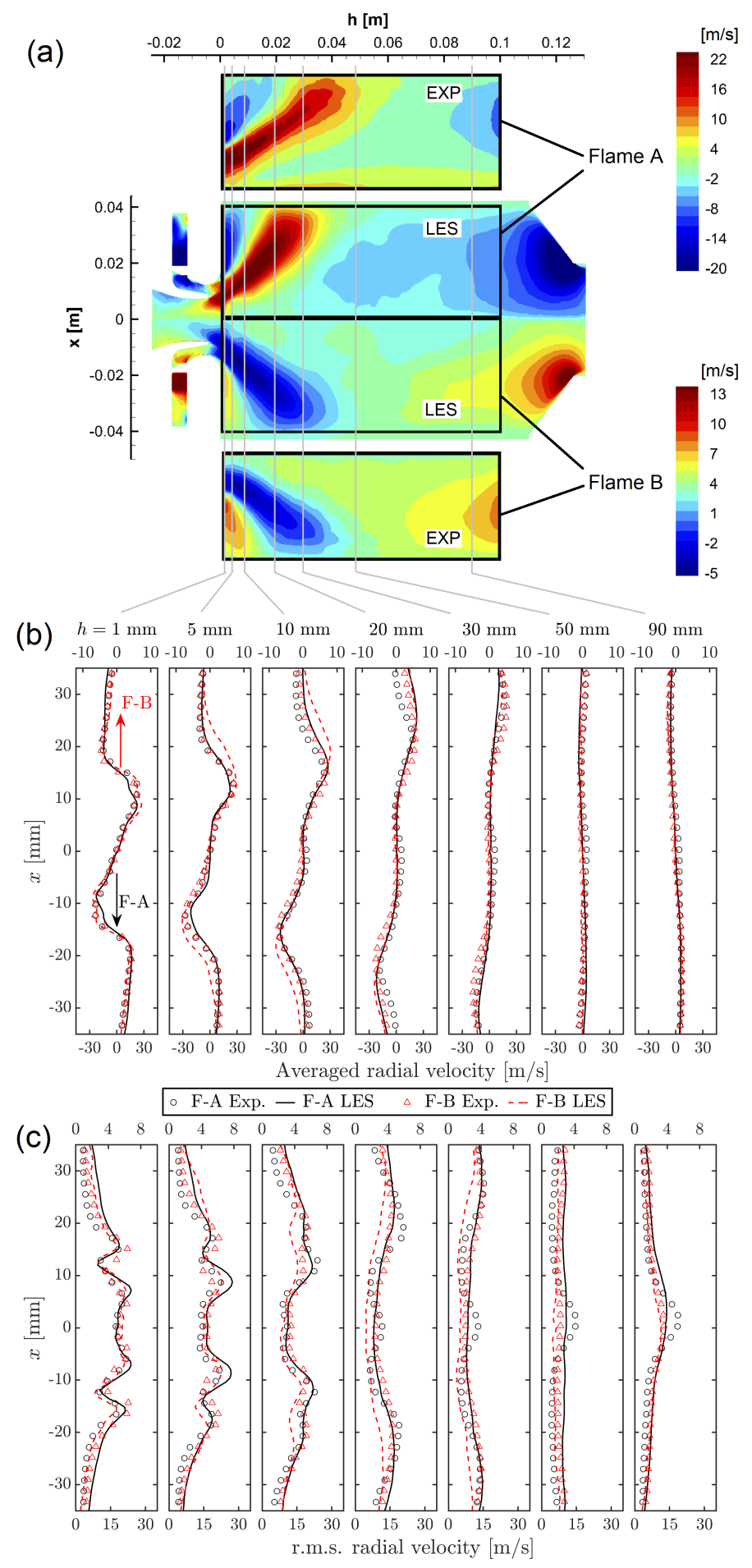

Fig. 6: Comparison of radial velocity for flames A and B: (a) mid-plane contour, (b) averaged and (c) r.m.s profiles at different axial locations. Symbols: experimental data. Lines: LES results. 


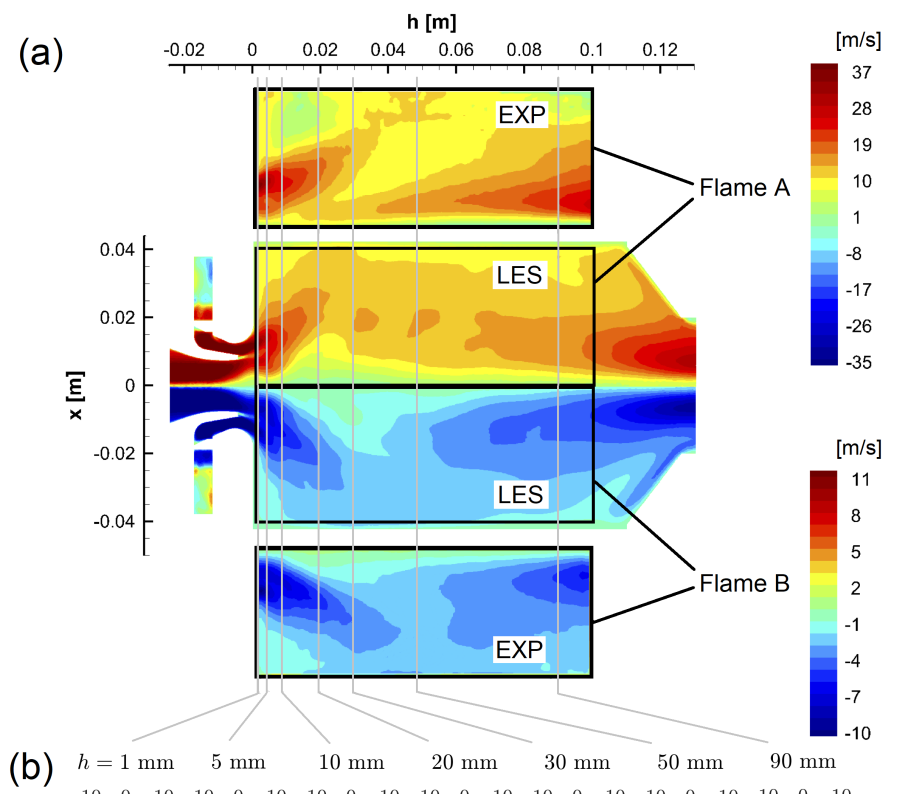

(b)
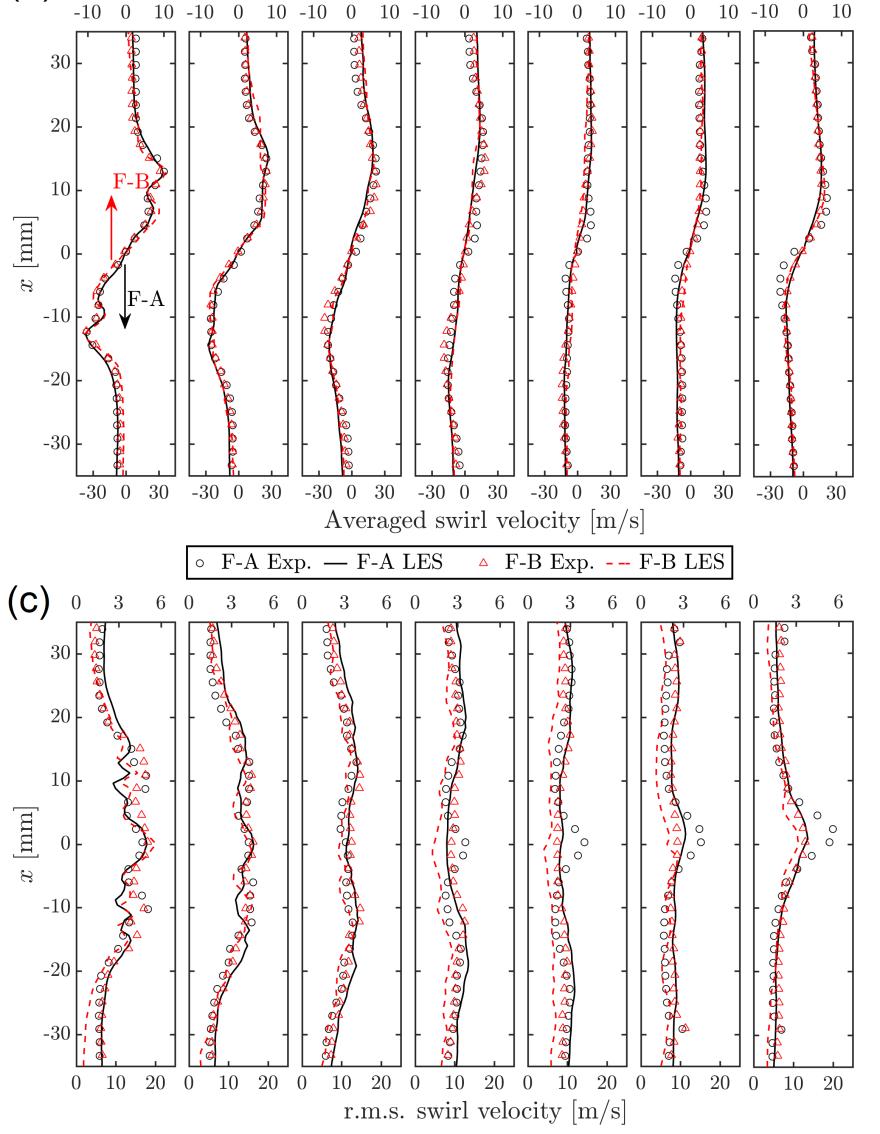

Fig. 7: Comparison of swirl velocity for flames A and B: (a) mid-plane contour, (b) averaged and (c) r.m.s profiles at different axial locations. Symbols: experimental data. Lines: LES results. 
Out-of-plane velocity $[\mathrm{m} / \mathrm{s}]$

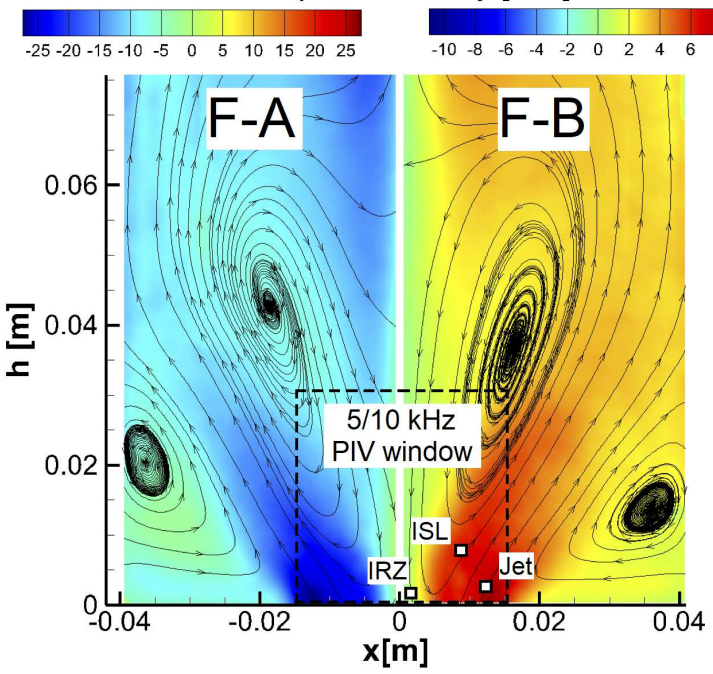

Fig. 8: Time averaged mid-plane streamlines overlaid on the out-ofplane velocity contours for flames A (left) and B (right). Dash lines mark the window for the high-repetition rate $(5 / 10 \mathrm{kHz})$ PIV measurements [21, 26]. (a) Probe-Jet

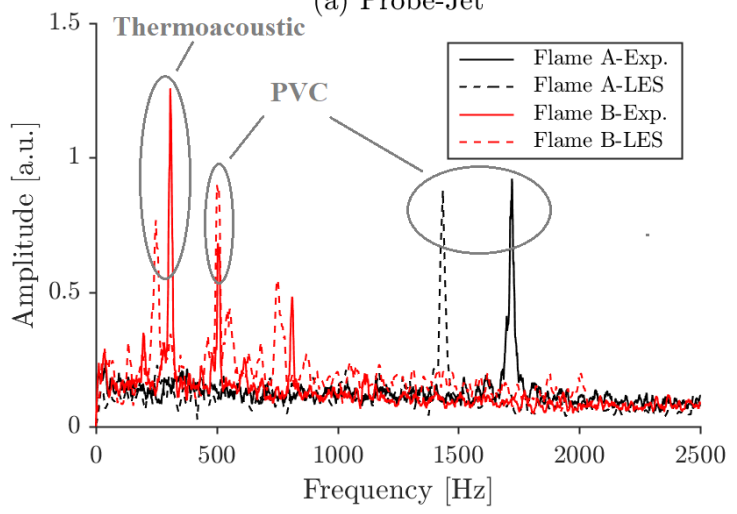

(b) Probe-ISL

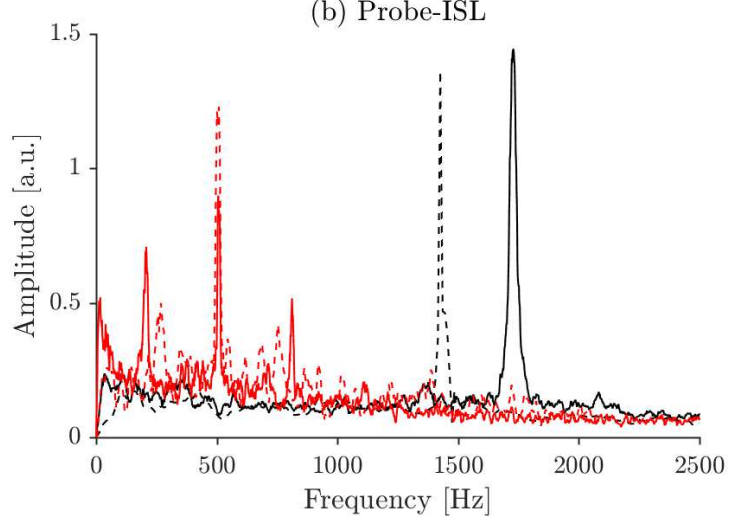

(c) Probe-IRZ

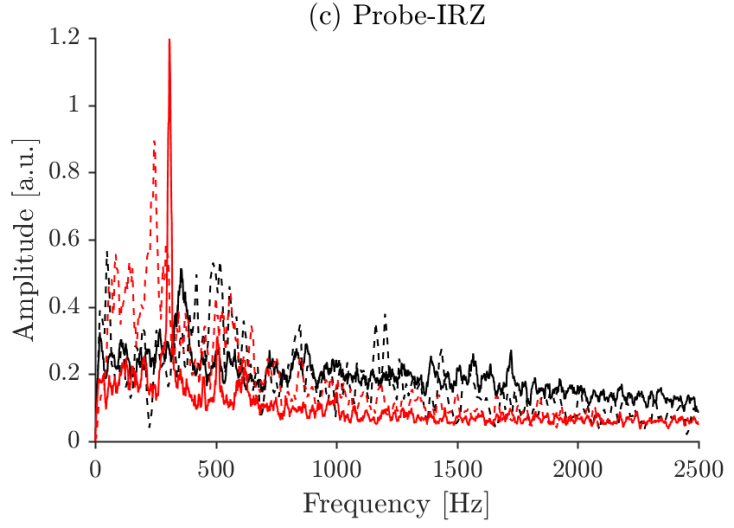

Fig. 9: Comparison of axial velocity spectra obtained from the experiments [21]26] (solid) and present LES (dashed) for Flame A (black) and $\mathrm{B}$ (red). The monitoring points are located in (a) the swirling jet (b) inner shear layer (ISL) and (c) inner recirculation zone (IRZ) as marked in Fig. 8 


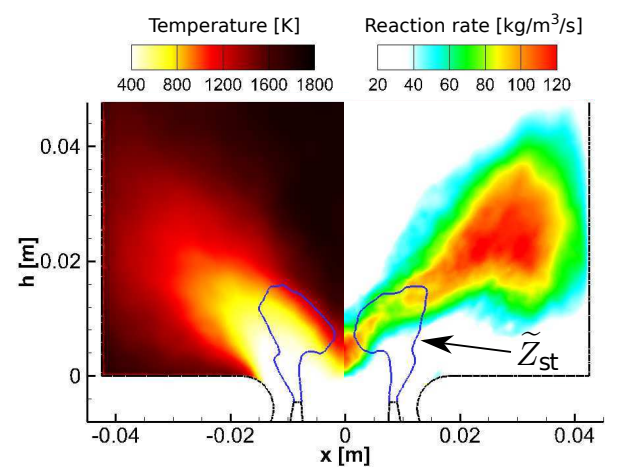

(a) Flame A

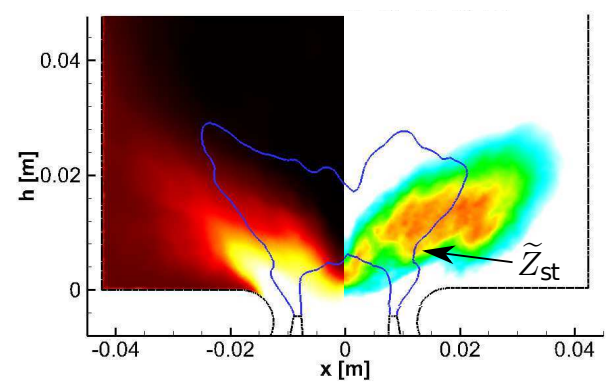

(b) Flame B

Fig. 10: Mid-plane contours of computed mean temperature (left) and reaction rate of progress variable (right) for (a) the stable flame A and (b) the unstable flame B. The iso-line corresponds to stoichiometric mixture fraction. 

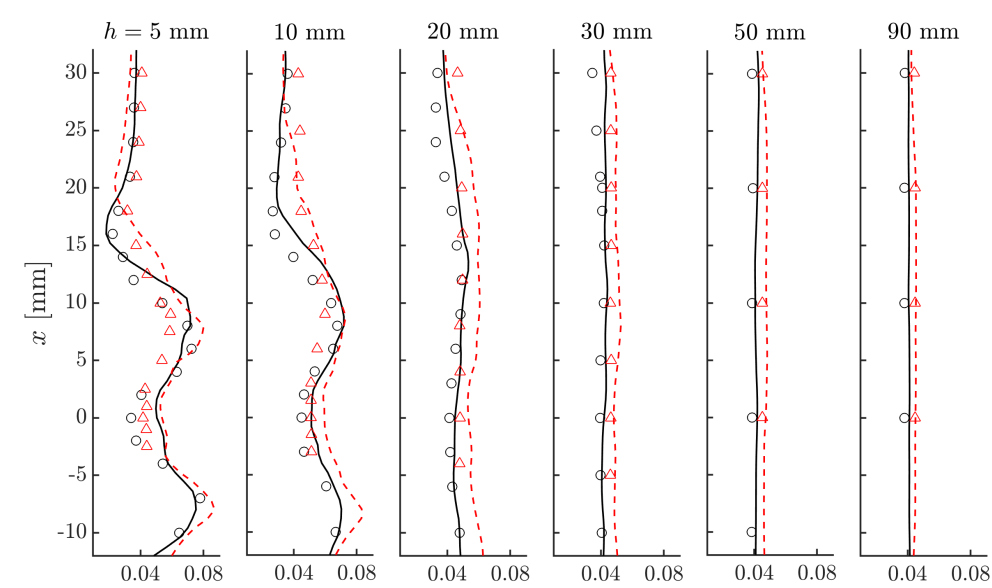

(a) Averaged mixture fraction
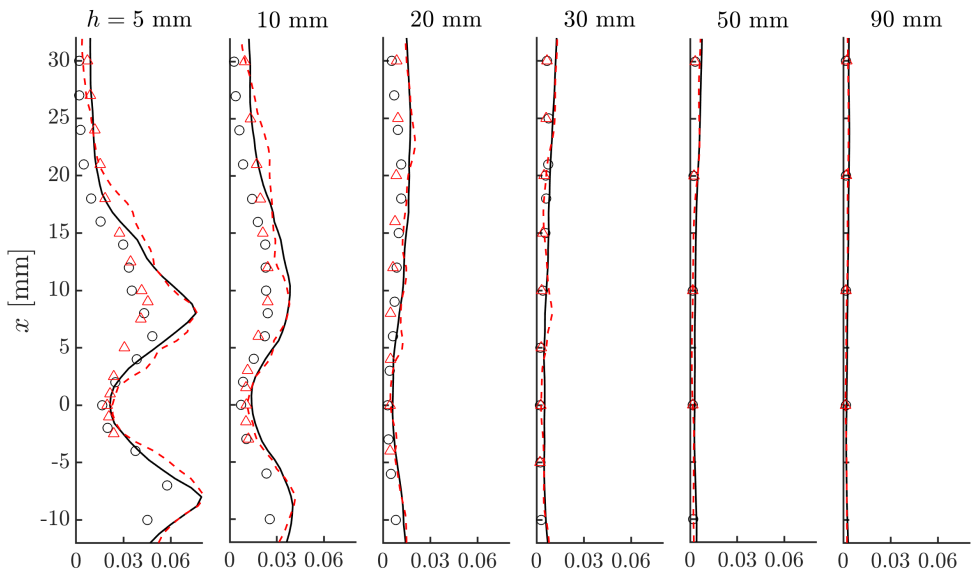

(b) r.m.s. mixture fraction

Fig. 11: Comparison of (a) averaged and (b) r.m.s. mixture fraction profiles for flames A (black) and B (red) at different axial positions. Symbols: experimental data. Lines: LES results. 


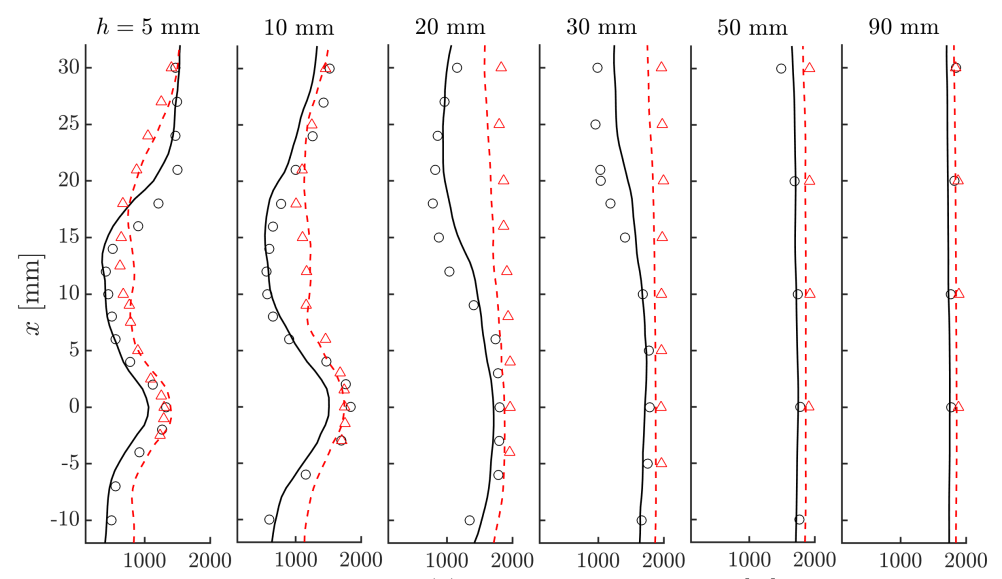

(a) Averaged temperature $[\mathrm{K}]$

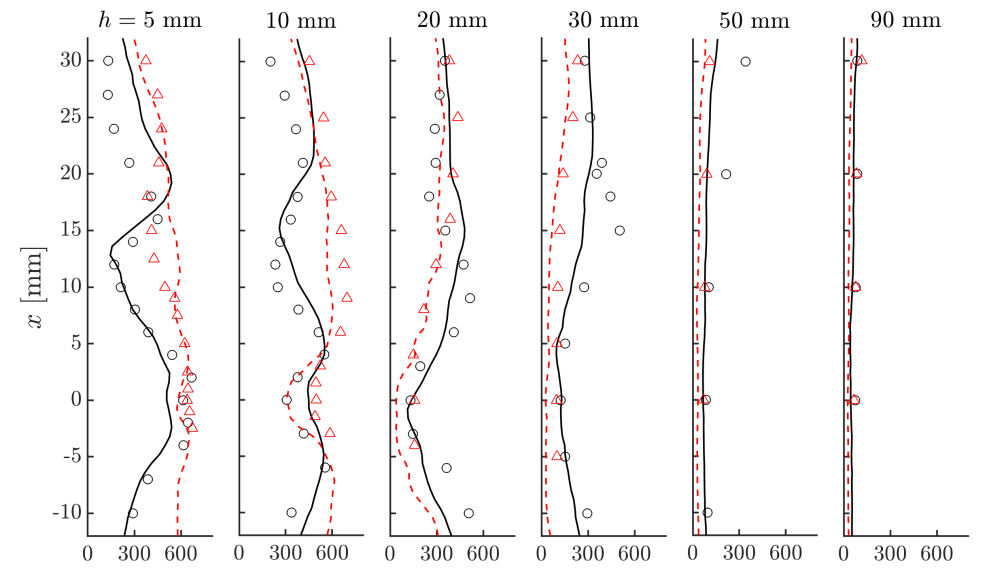

(b) r.m.s. temperature $[\mathrm{K}]$

Fig. 12: Comparison of (a) averaged and (b) r.m.s. temperature profiles for flames A (black) and B (red) at different axial positions. Symbols: experimental data. Lines: LES results. 

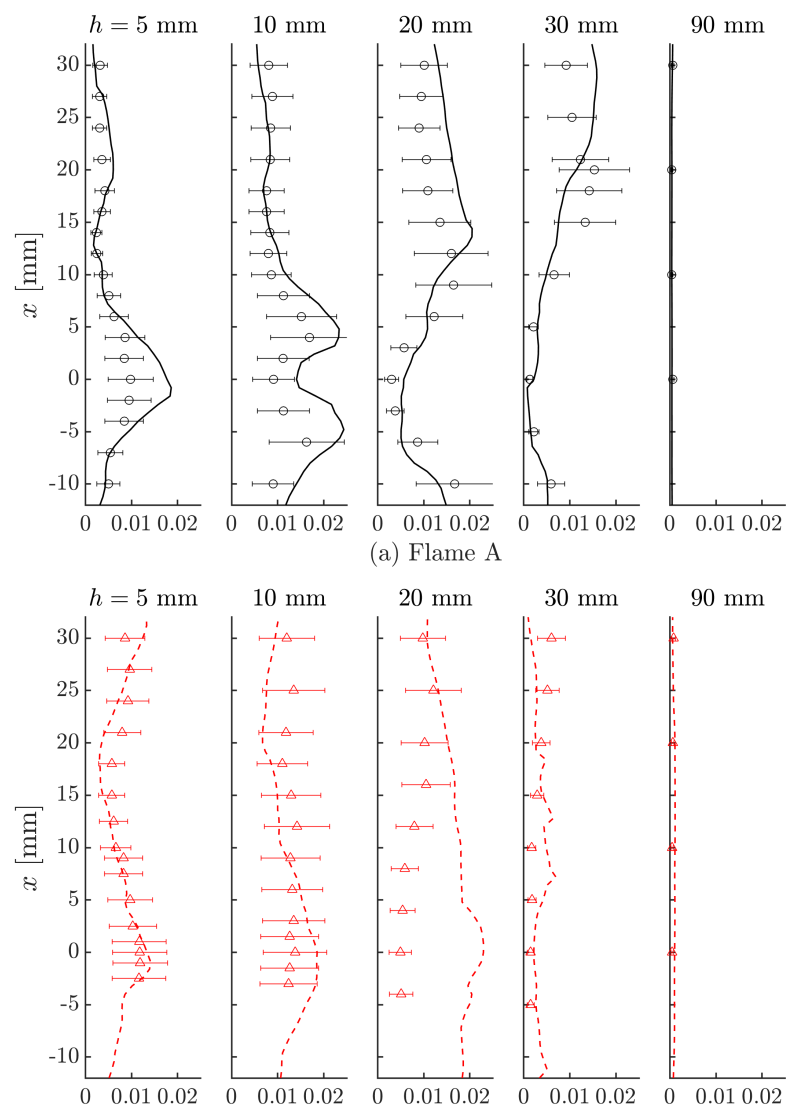

(a) Flame A

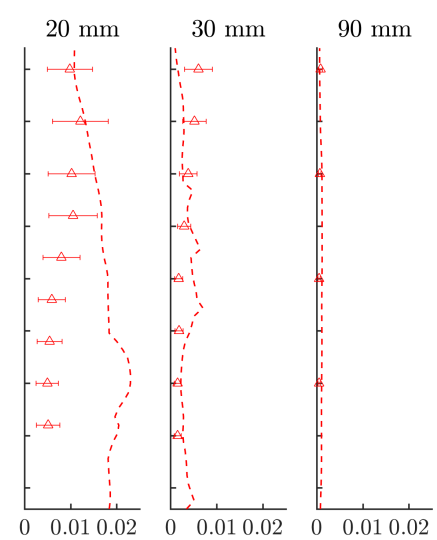

(b) Flame B

Fig. 13: Mean $\mathrm{CO}$ mass fraction profiles at different axial positions for (a) the stable flame A and (b) the unstable flame B. Symbols: experimental data. Lines: LES results. 

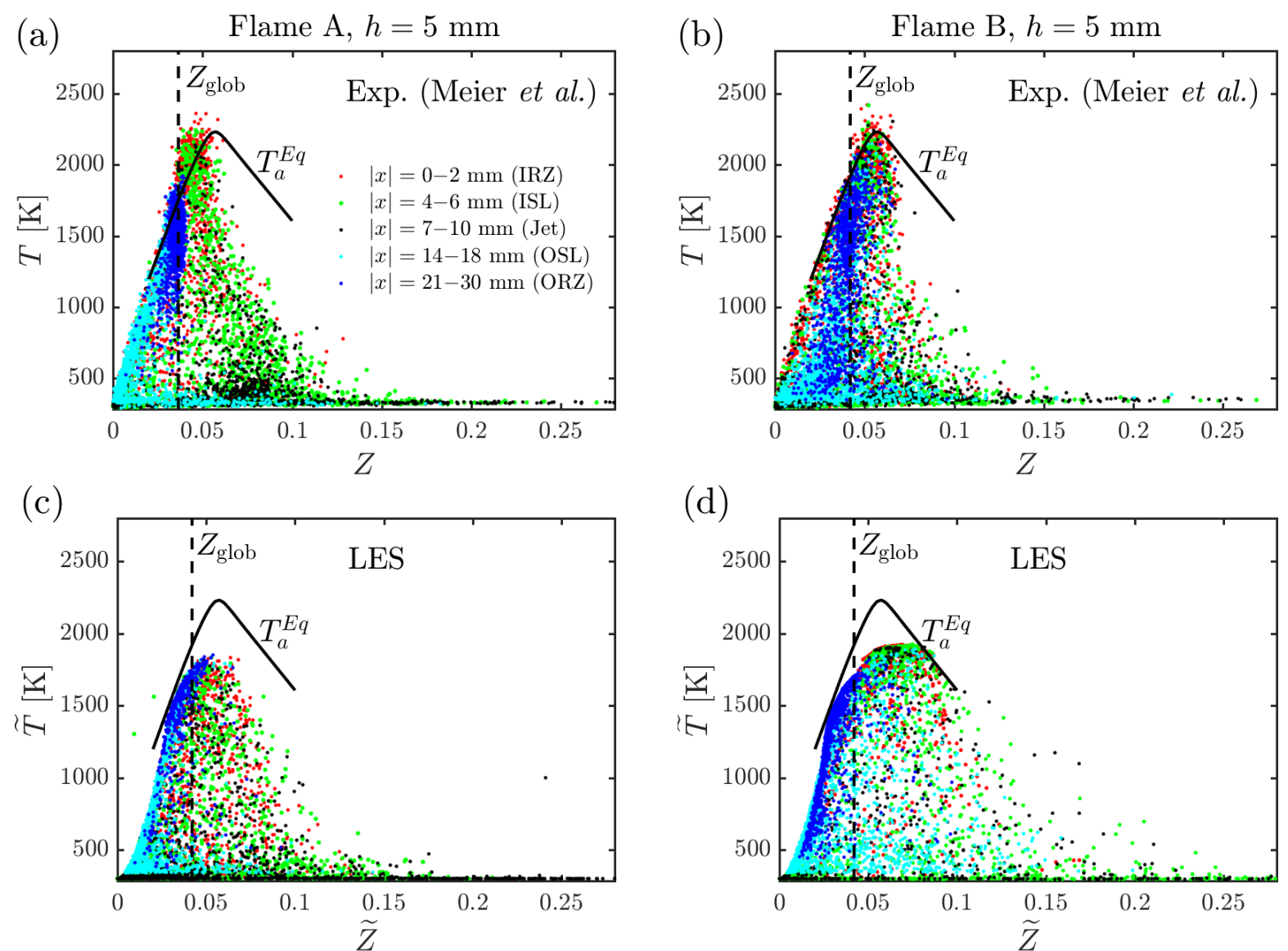

Fig. 14: Scatter plots of temperature vs. mixture fraction at $h=5 \mathrm{~mm}$ obtained from experiments (top) and LES (bottom) for Flame A (left) and B (right). The solid line corresponds to the adiabatic equilibrium temperature and the dash line denotes the global mixture fraction (see Table 1 . 

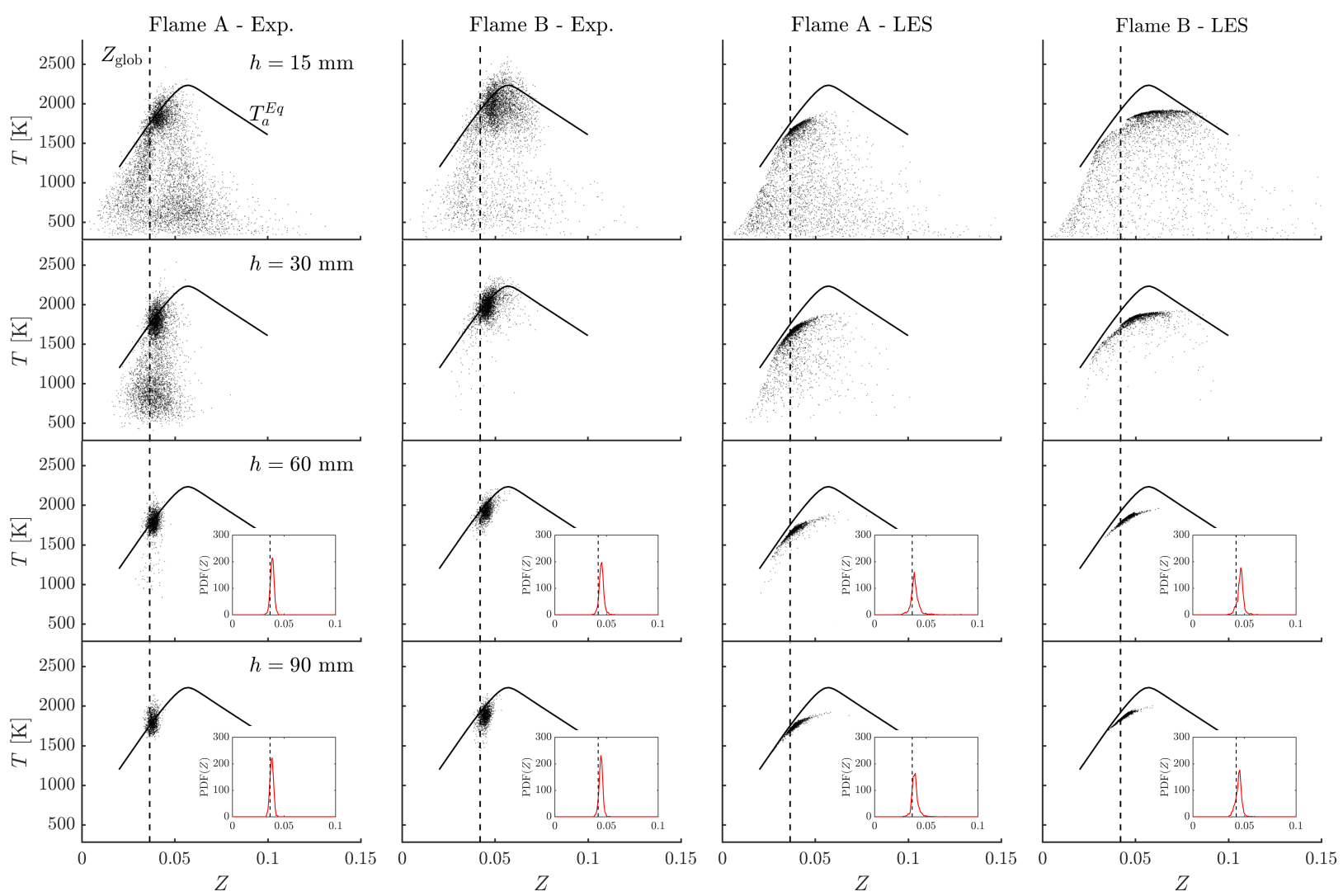

Fig. 15: Scatter plots of temperature vs. mixture fraction obtained from experiments and LES at different axial positions. The PDF of mixture fraction is shown for $h=60$ and $90 \mathrm{~mm}$.

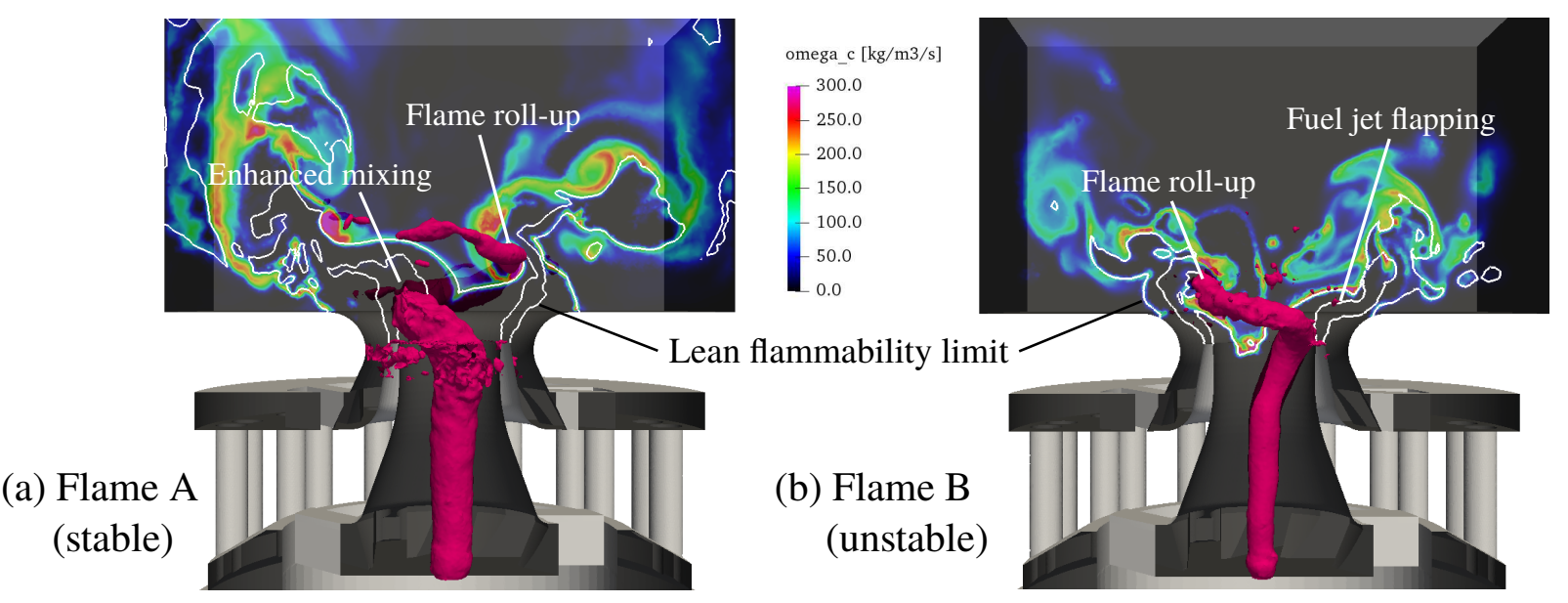

Fig. 16: Typical snapshot of the filtered reaction rate contour in the mid-plane with 3D presentation of the PVC. The mixture fraction iso-line (thin white line) represents the lean flammability limit. 


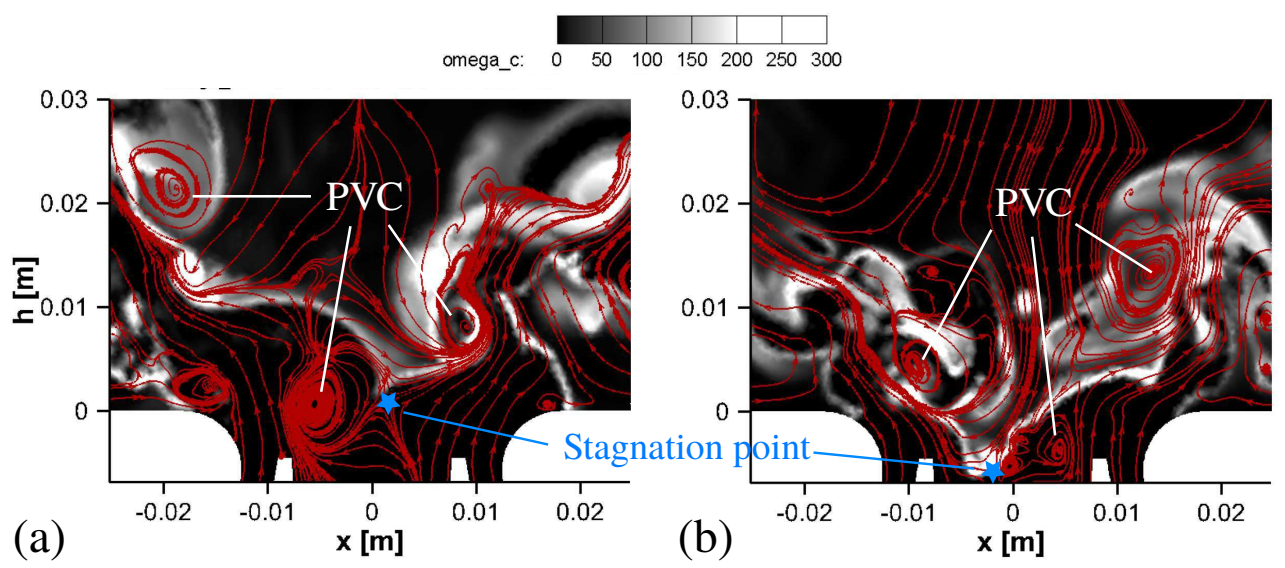

Fig. 17: Mid-plane flow stagnation points and streamlines overlaid on filtered reaction rate contour for the same snapshot shown in Fig. 16

(a) Flame A (stable)
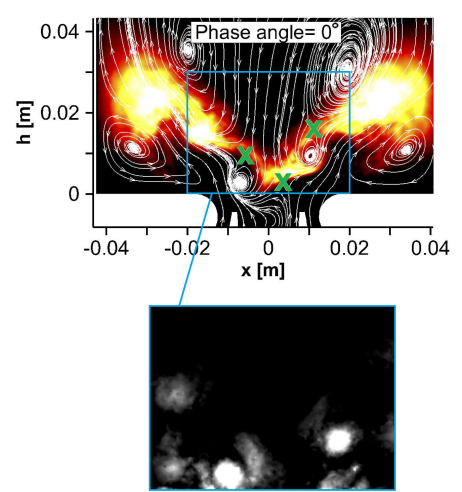

X Stagnation points

(b) Flame B (unstable)
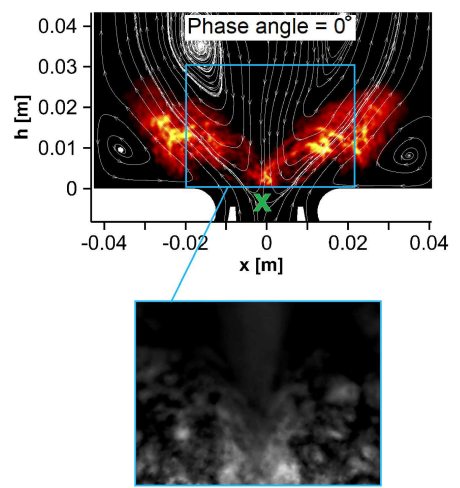

$[\mathrm{kg} / \mathrm{m} 3 / \mathrm{s}]$

omega_c : $0 \begin{array}{lllllll}0 & 20 & 40 & 60 & 80 & 100 & 120\end{array}$
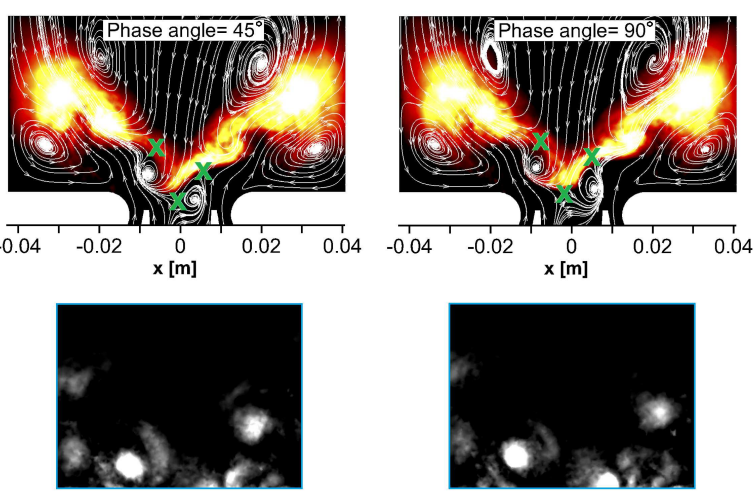

Swirl strength:
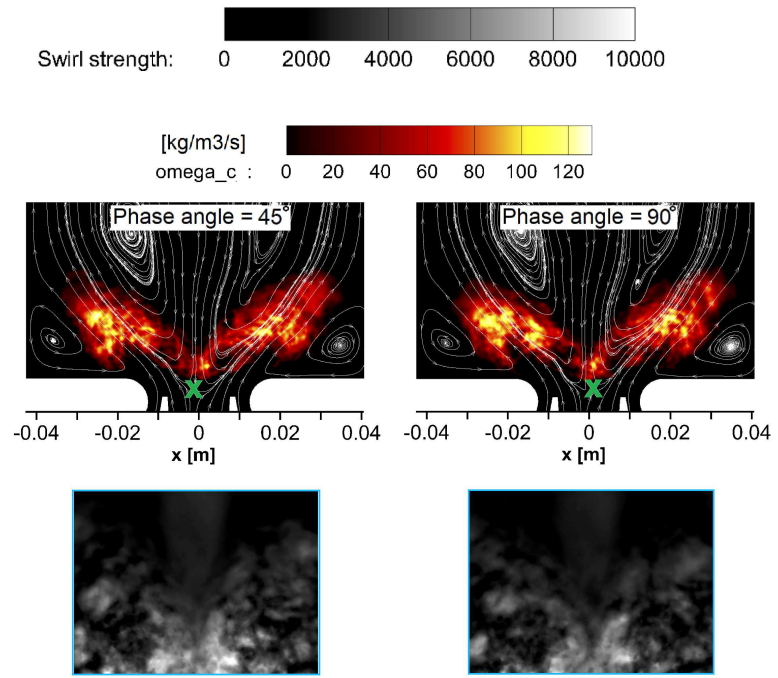

Swirl strength:

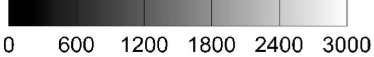

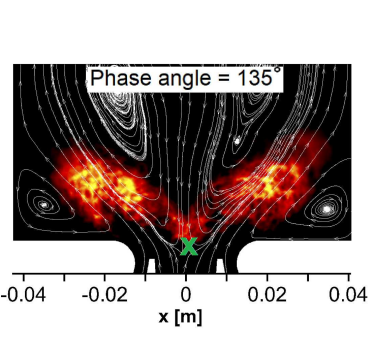
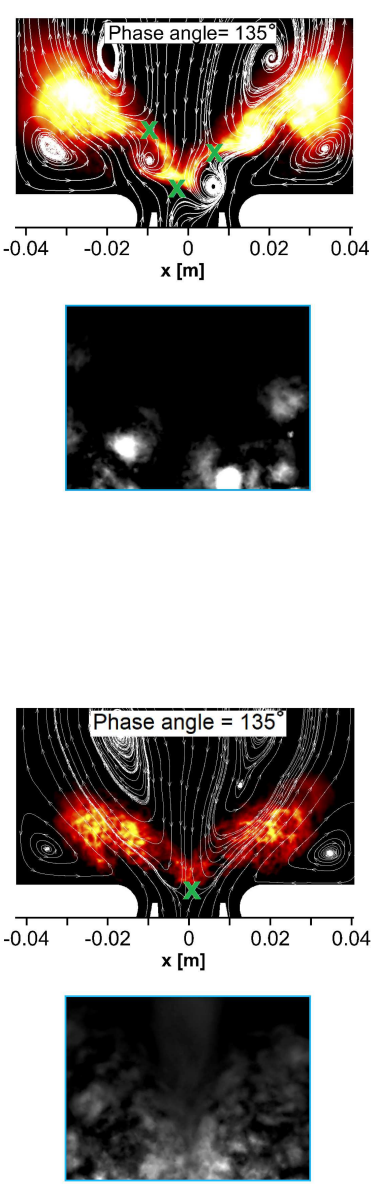

Fig. 18: Phase-averaged streamlines overlaid on filtered reaction rate contour for flames (a) A and (b) B. The swirl strength factor [65] is plotted in the enlarged windows. 


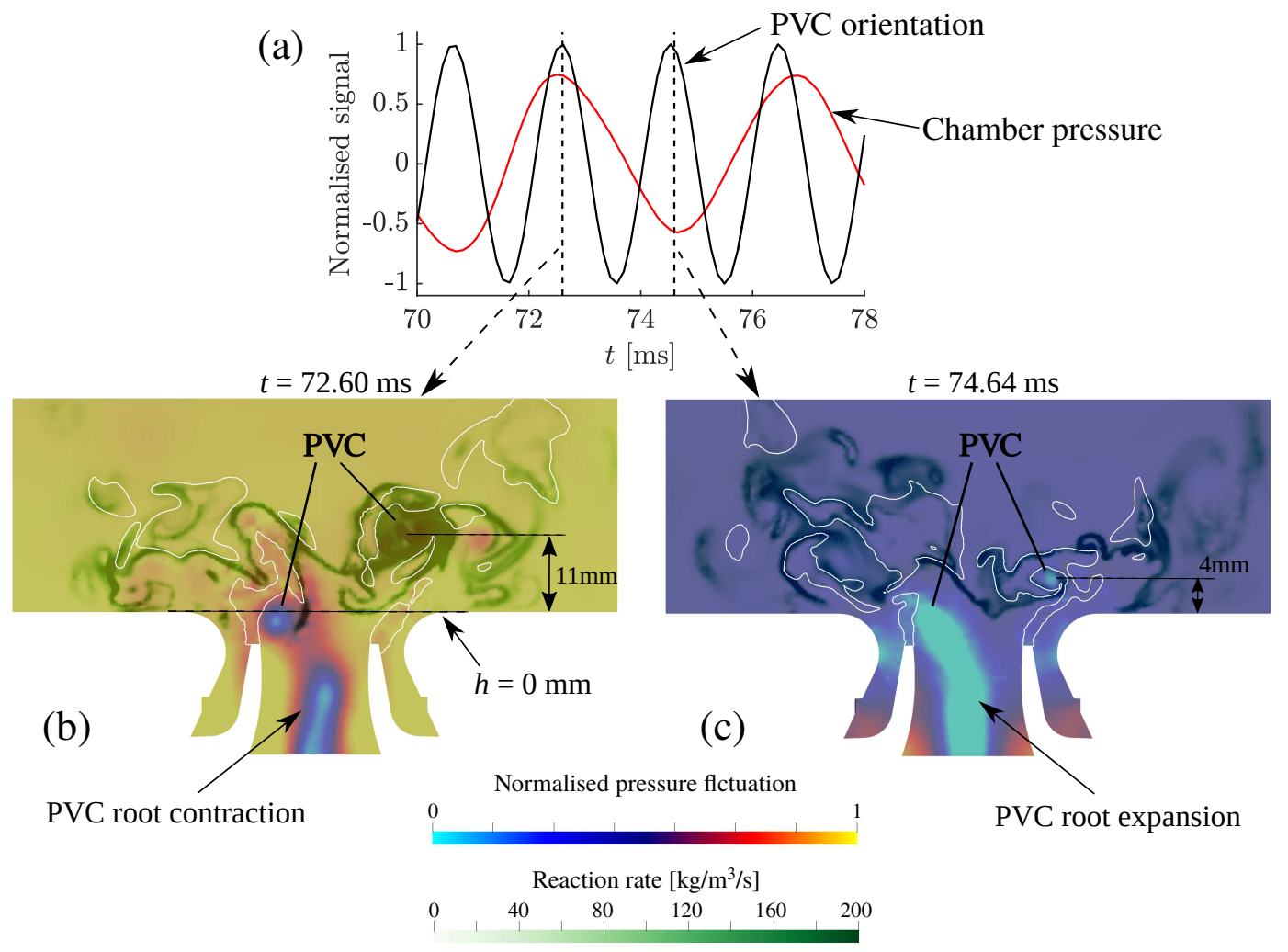

Fig. 19: (a)Temporal variation of the PVC orientation and combustion chamber pressure for flame B. Representative PVC behaviours are shown for the (b) minimum and (c) maximum chamber pressure during a thermoacoustic oscillation cycle using mid-plane contours of reaction rate overlaid on pressure. The white lines denote $\widetilde{Z}=0.07$. 
(a)

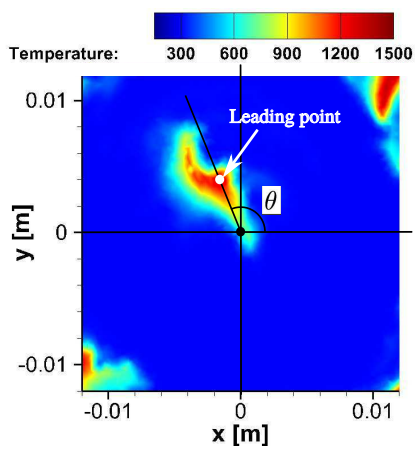

(b)

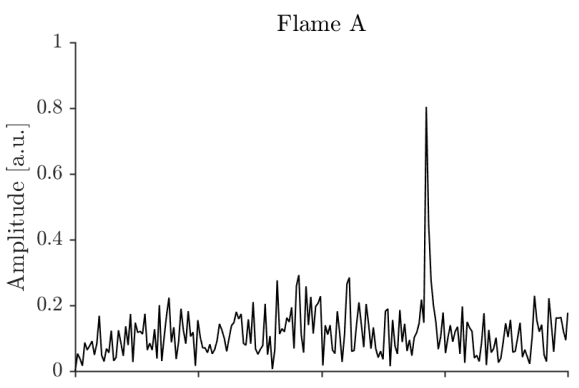

(c)

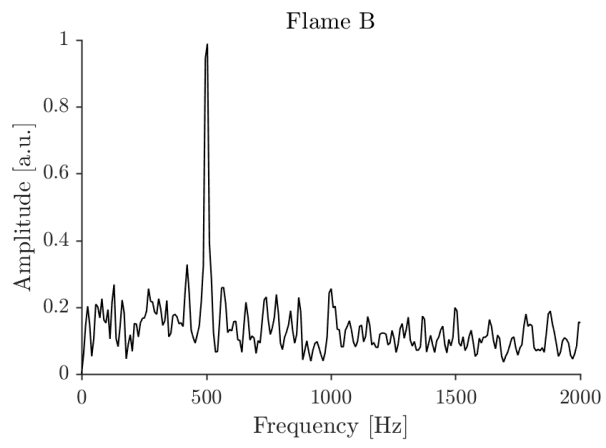

Fig. 20: Azimuthal movement of the flame leading point: (a) transverse-plane contour of temperature at the lift-off height. FFT of $\theta^{\prime}$ for flames (b) A and (c) B. 


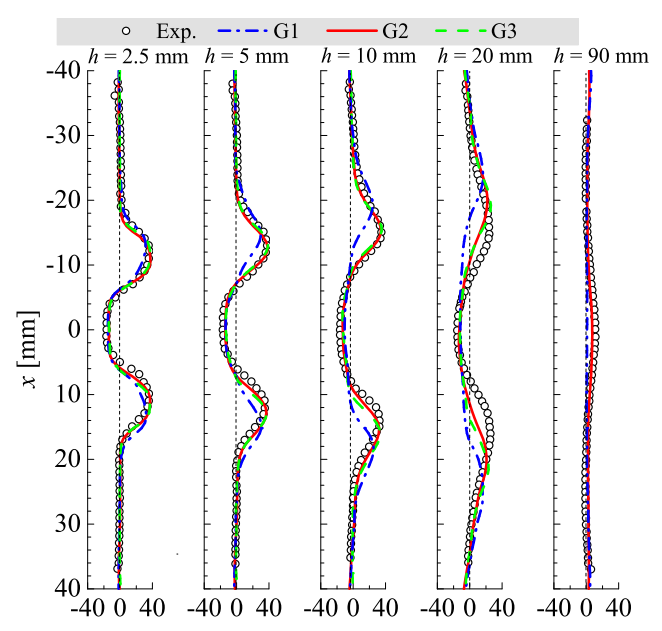

(a) Averaged axial velocity $[\mathrm{m} / \mathrm{s}]$

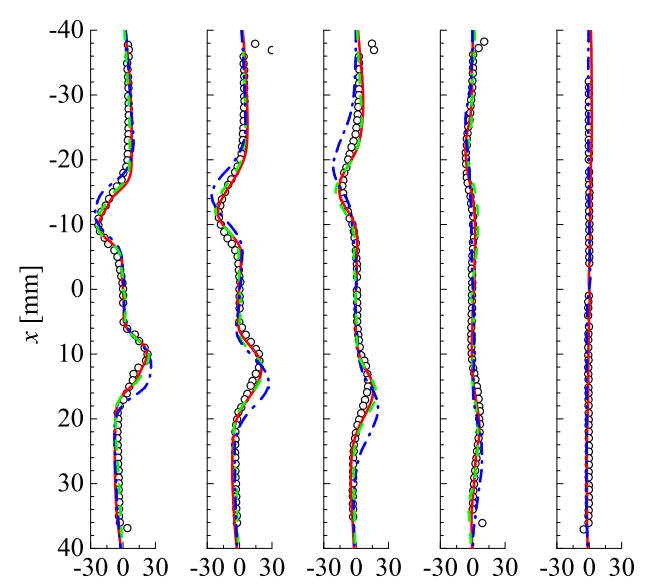

(c) Averaged radial velocity $[\mathrm{m} / \mathrm{s}]$

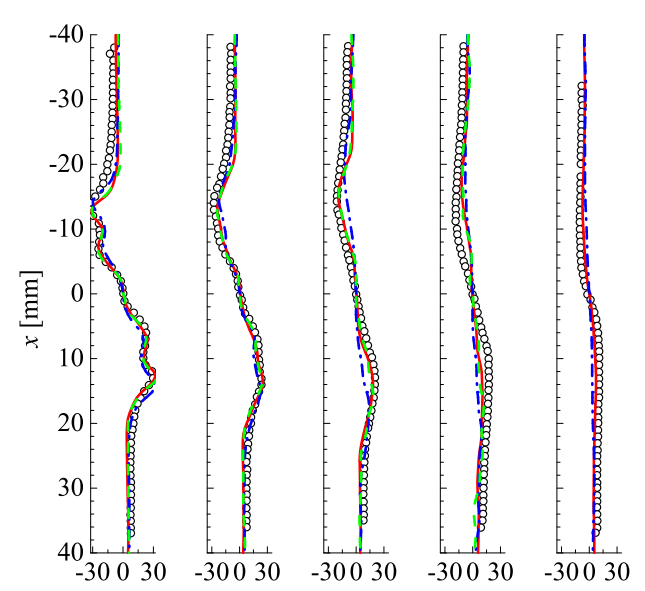

(e) Averaged swirl velocity $[\mathrm{m} / \mathrm{s}]$

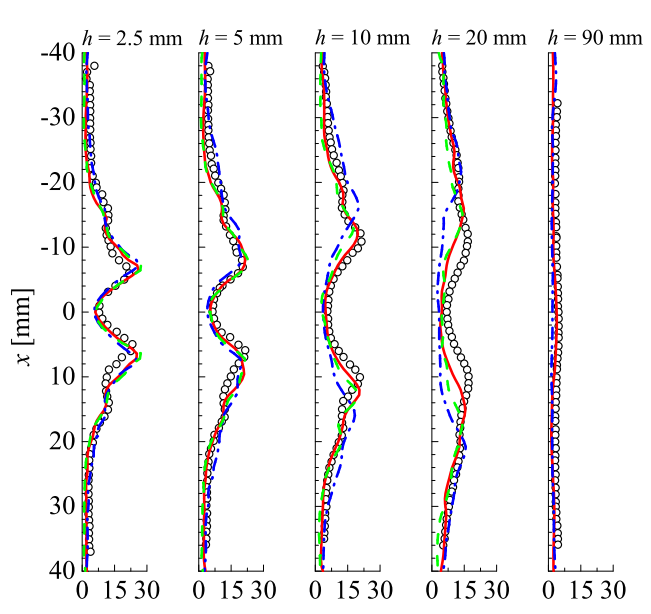

(b) r.m.s. axial velocity $[\mathrm{m} / \mathrm{s}]$
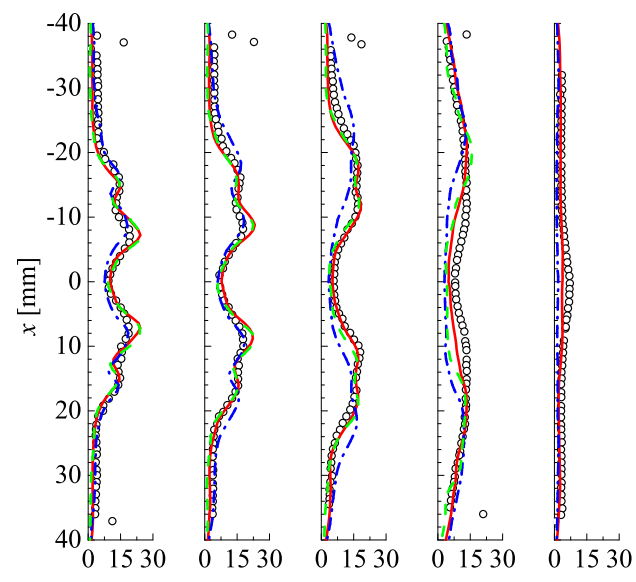

(d) r.m.s. radial velocity $[\mathrm{m} / \mathrm{s}]$
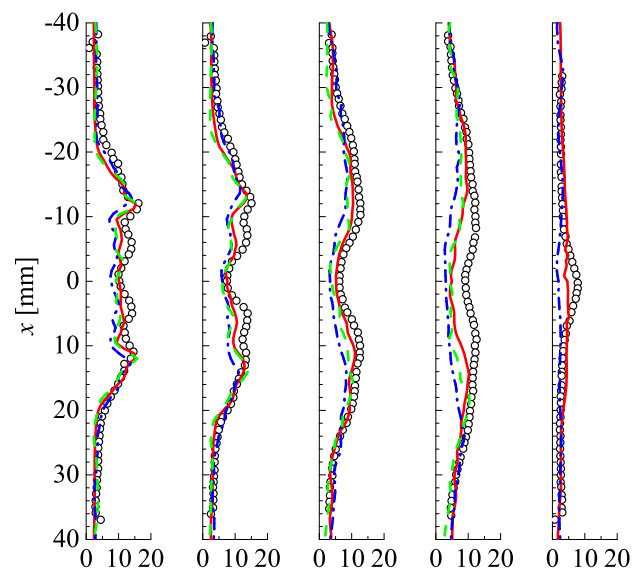

(f) r.m.s. swirl velocity $[\mathrm{m} / \mathrm{s}]$

Fig. A.1: Cold flow comparison of axial, radial and swirl velocity radial profiles for five streamwise locations. Symbols: measurements [67]. Lines: LES using three different grid resolutions. 

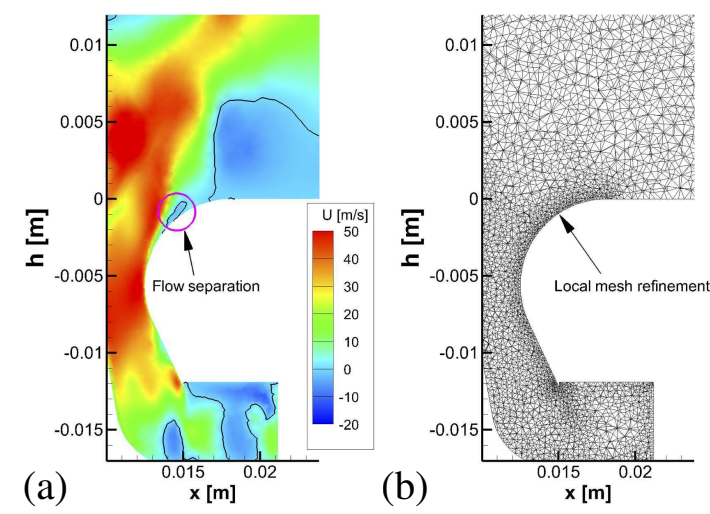

Fig. A.2: (a) Mid-plane axial velocity contours and (b) local mesh refinement for flow separation prediction at the outer air nozzle exit. The black line on the left denotes zero-velocity.

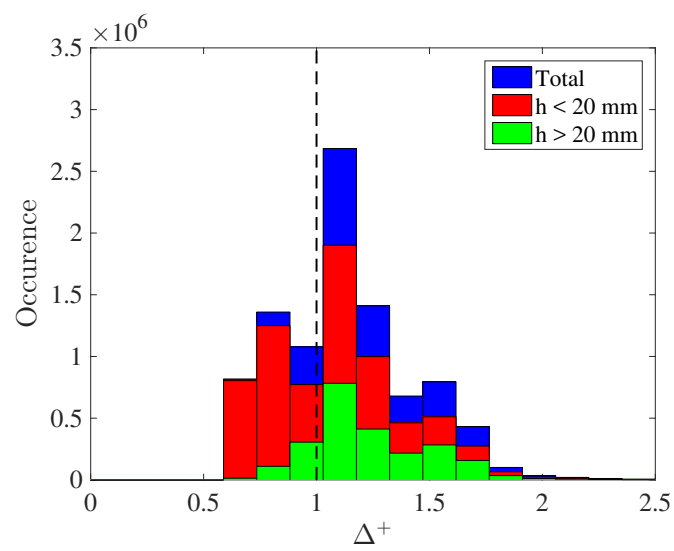

Fig. A.3: Histogram of the normalised filter size $\Delta^{+}$distribution for computational cells with $\overline{\dot{\omega}_{\mathrm{c}}^{*}}>0$. The dashed line highlights the filter size equal to the reference laminar flame thickness. 